Article

\title{
Stereoselective Formation of Substituted 1,3-Dioxolanes through a Three-Component Assembly during the Oxidation of Alkenes with Hypervalent Iodine(III)
}

\author{
Mio Shimogaki, Morifumi Fujita * and Takashi Sugimura \\ Graduate School of Material Science, University of Hyogo, Kohto, Kamigori, Hyogo 678-1297, Japan; \\ E-Mails: rk14v001@stkt.u-hyogo.ac.jp (M.S.); sugimura@sci.u-hyogo.ac.jp (T.S.) \\ * Author to whom correspondence should be addressed; E-Mail: fuji@sci.u-hyogo.ac.jp; \\ Tel.: +81-791-58-0170; Fax: +81-791-58-0115.
}

Academic Editors: Wesley Moran and Arantxa Rodríguez

Received: 14 August 2015 / Accepted: 11 September 2015 / Published: 17 September 2015

\begin{abstract}
Stereoselective formation of substituted 1,3-dioxolanes was achieved through an assembly of three components: alkene, carboxylic acid and silyl enol ether. The reaction proceeded via stereospecific generation of a 1,3-dioxolan-2-yl cation intermediate during oxidation of alkene substrates with hypervalent iodine. The stereoselective trapping of the cation intermediate with silyl enol ether completed the formation of the dioxolane product.
\end{abstract}

Keywords: hypervalent iodine; oxidation; dioxolane; dioxolanyl cation; stereoselective synthesis

\section{Introduction}

Oxidative difunctionalization of alkenes with hypervalent iodine reagents is one of the many attractive and powerful transformations, as well as halogenation, oxygenation, amidation and arylation (alkylation), for forming useful synthetic intermediates [1-44]. During oxidation, two nucleophiles are attached to the carbon-carbon double bond (Figure 1a) [20-26]. This approach has also been applied to the regio- and stereoselective preparation of heterocyclic compounds. An alkene substrate containing a simple intramolecular nucleophile provides a heterocyclic product (Figure 1b) [27-40]. When a bidentate nucleophile such as urea is introduced to the alkene substrate, bicyclic products are obtained (Figure 1c) [41-44]. To expand upon the diverse structures of the resulting heterocyclic products, we 
postulated that intermolecular heterocyclic formation would be achieved using an external bidentate nucleophile (Figure 1d) [45-47]. To demonstrate this concept, we focused on the 1,3-dioxolan-2-yl cation intermediate $[48,49]$ generated during hypervalent-iodine-mediated Prévost and Woodward reactions [50-58]. In these reactions, a carboxylic acid acts as a bidentate nucleophile; however, the heterocyclic intermediate changes to a ring-opening product. To maintain the heterocyclic structure of dioxolane, a subsequent nucleophilic attack should occur at the 2-position to give a dioxolane product in stable form $[59,60]$. Herein, we describe the stereoselective preparation of substituted dioxolanes through condensation of three components: alkene, carboxylic acid and carbon nucleophile during the hypervalent-iodine-mediated Prévost and Woodward reaction. The stereoselectivity must be controlled by two steps: the oxidative formation of a 1,3-dioxolan-2-yl cation intermediate and its nucleophilic trapping (Figure 1d).

(a) external nucleophiles

$$
\mathrm{R} \leftrightharpoons \mathrm{R}^{\prime} \underset{\mathrm{Nu}-\mathrm{H}}{\stackrel{\mathrm{X}-\mathrm{I}-\mathrm{X}}{\mathrm{Ar}}} \stackrel{\mathrm{X}_{\mathrm{X}}^{\mathrm{Nu}}}{\mathrm{R}^{\prime}}
$$

(b) intramolecular nucleophile

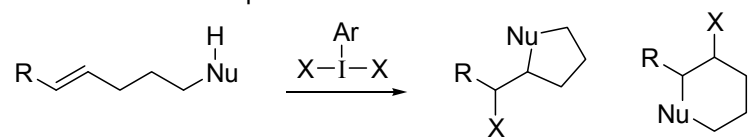

(c) intramolecular bidentate nucleophile

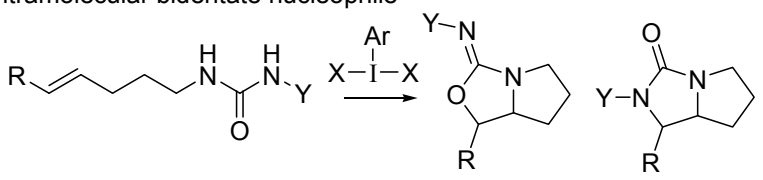

(d) external bidentate nucleophile: This work

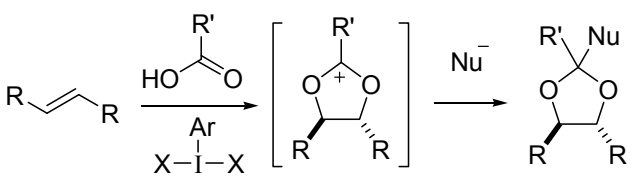

Figure 1. Difunctionalization of alkenes with hypervalent iodine(III) using (a) external nucleophiles; (b) intramolecular nucleophile; (c) intramolecular bidentate nucleophile; and (d) external bidentate nucleophile.

\section{Results and Discussion}

We initially selected cis-4-octene (1a) as a model substrate to test the feasibility of this transformation (Table 1). The reaction of 1a with (diacetoxyiodo)benzene was initiated by injection of boron trifluoride diethyl etherate at $-80{ }^{\circ} \mathrm{C}$ into a dichloromethane solution containing AcOH. After warming to $-40{ }^{\circ} \mathrm{C}$, dimethyl ketene silyl acetal $2 \mathbf{a}$ was added to the reaction mixture to produce a dioxolane product 3a as a single diastereomer (Table 1, entries 1-3). The yield of 3a was affected by the amount of nucleophiles (acetic acid and 2a), as well as the temperature of reaction termination; the maximum yield was $62 \%$ (entry 3 ). In the ${ }^{13} \mathrm{C}-\mathrm{NMR}$ of $\mathbf{3 a}$, ten signals were observed. This indicates that the adduct 3a has $C_{\mathrm{s}}$ symmetry (meso configuration). Moreover, the stereochemistry of 3a was determined to be the (2r)-configuration by nOe between $H-4,5$ and the dimethyl moiety (the illustration in Table 1), which was observed in an NMR NOESY experiment (Supplementary Materials (SM)). The 
corresponding chiral racemic isomer $\mathbf{3 b}$ was selectively obtained from the reaction with trans-4-octene $\mathbf{1 b}$ (entry 4). In the ${ }^{13} \mathrm{C}-\mathrm{NMR}$ spectrum of $\mathbf{3 b}$, fifteen signals were detected.

Table 1. Reaction of cis-4-octene $\mathbf{1 a}$ and trans-4-octene $\mathbf{1 b}{ }^{\mathrm{a}}$.

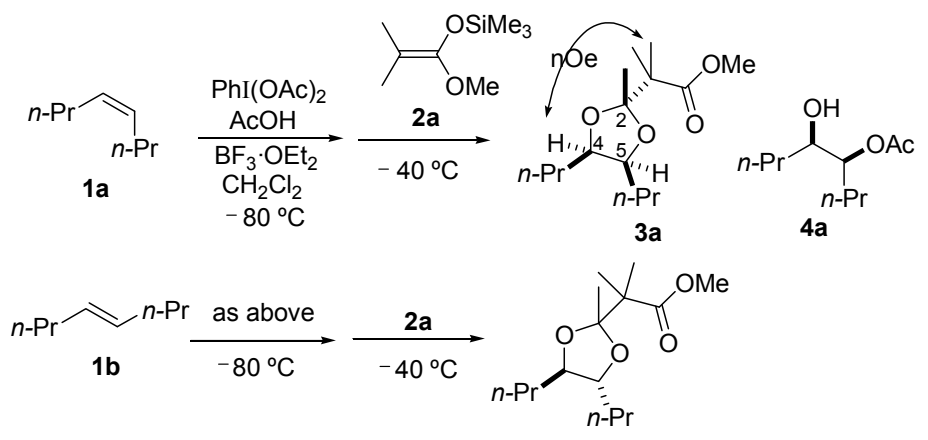

\begin{tabular}{cccccc} 
& \multicolumn{5}{c}{ 3b } \\
\hline Entry & $\mathbf{1}$ & AcOH (mmol) & 2a (mmol) & Quench Temp. & Yield (\%) \\
\hline 1 & $\mathbf{1 a}$ & 1.7 & 0.5 & $\mathrm{rt}$ & $\mathbf{3 a}, 2 ; \mathbf{4 a}, 58$ \\
2 & $\mathbf{1 a}$ & 0.5 & 1.5 & $\mathrm{rt}$ & $\mathbf{3 a}, 46$ \\
3 & $\mathbf{1 a}$ & 0.5 & 1.5 & $-30{ }^{\circ} \mathrm{C}$ & $\mathbf{3 a}, 62$ \\
4 & $\mathbf{1 b}$ & 0.5 & 1.5 & $-30{ }^{\circ} \mathrm{C}$ & $\mathbf{3 b}, 48$ \\
\hline
\end{tabular}

${ }^{a}$ Reaction was initiated at $-80{ }^{\circ} \mathrm{C}$ in the presence of $1(0.32 \mathrm{mmol}), \mathrm{PhI}(\mathrm{OAc})_{2}(0.40 \mathrm{mmol})$ and $\mathrm{BF}_{3} \cdot \mathrm{OEt}_{2}$ (0.8 mmol) in $\mathrm{CH}_{2} \mathrm{Cl}_{2}(4 \mathrm{~mL})$ and $\mathrm{AcOH}$. Then, $2 \mathbf{a}$ was added at $-40{ }^{\circ} \mathrm{C}$.

The stereospecific and stereoselective formation of the substituted dioxolane products can be explained by reaction pathways involving the 1,3-dioxolan-2-yl cation intermediate (Scheme 1). This intermediate was generated by participation of the neighboring acetoxy group in the oxidation of the alkene substrate with hypervalent iodine in the presence of $\mathrm{AcOH}$. The neighboring group participation may cause stereospecific formation of the dioxolanyl cation intermediate, i.e., the cis alkene 1a forms the meso cation and the trans-alkene $\mathbf{1 b}$ forms the chiral cation.

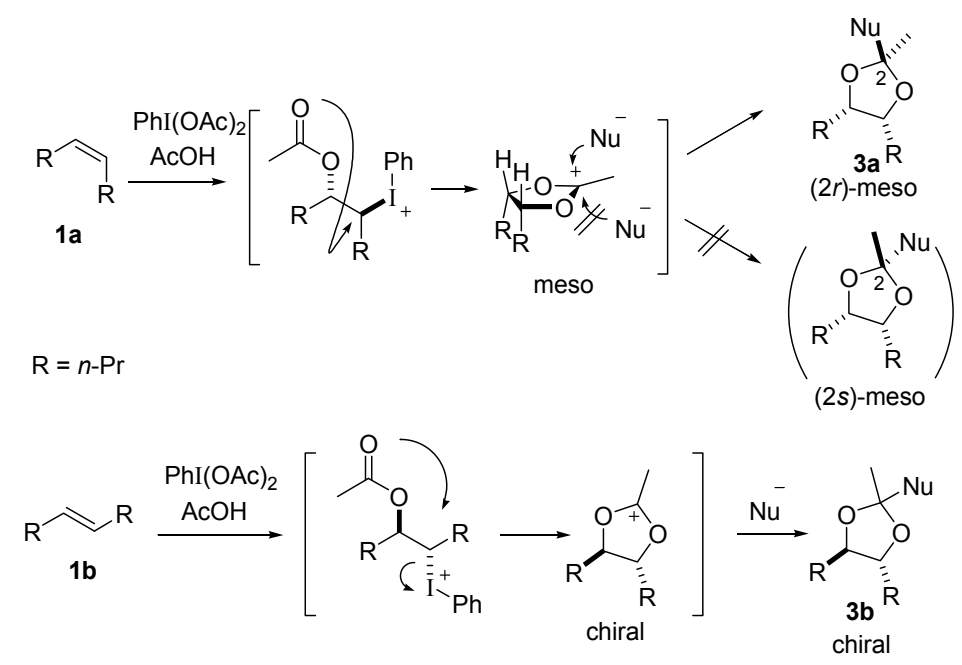

Scheme 1. Plausible pathway for stereoselective formation of dioxolane 3.

Stereoselectivity in nucleophilic trapping with $\mathbf{2 a}$ affects the stereochemical outcome of the dioxolane products. The meso cation generated from the cis alkene has diastereofaces for the nucleophilic addition. Thus, the diastereoselective formation of the trapping product requires stereoface control in the 
nucleophilic attack, as illustrated in Scheme 1. The actual diastereoselective formation of the (2r)-meso product starting from cis alkene may be attributed to the steric fence provided by the 4,5-substituents of the meso cation. Owing to the $C_{2}$ symmetry of the chiral cation generated from the trans-octene, the nucleophilic trapping of the chiral cation led to the chiral dioxolane product $\mathbf{3 b}$ as a single diastereomer without any stereocontrol during the nucleophilic trapping.

The proposed reaction pathway (Scheme 1) was also supported by control experiments without the addition of the trapping reagent $\mathbf{2 a}$ (Scheme 2). The acetoxyhydroxy products $\mathbf{4 a}$ and $\mathbf{4 b}$ were stereospecifically obtained from cis-octene 1a and trans-octene 1b, respectively; their stereochemical configurations were confirmed after transformation to diacetoxy products: meso form 5a and racemic form 5b. The stereochemical outcome obeyed that of a typical Woodward reaction: trapping of 1,3-dioxolan-2-yl cation with water at the 2-position and subsequent ring-opening reaction result in retention of configuration of the cation intermediate.

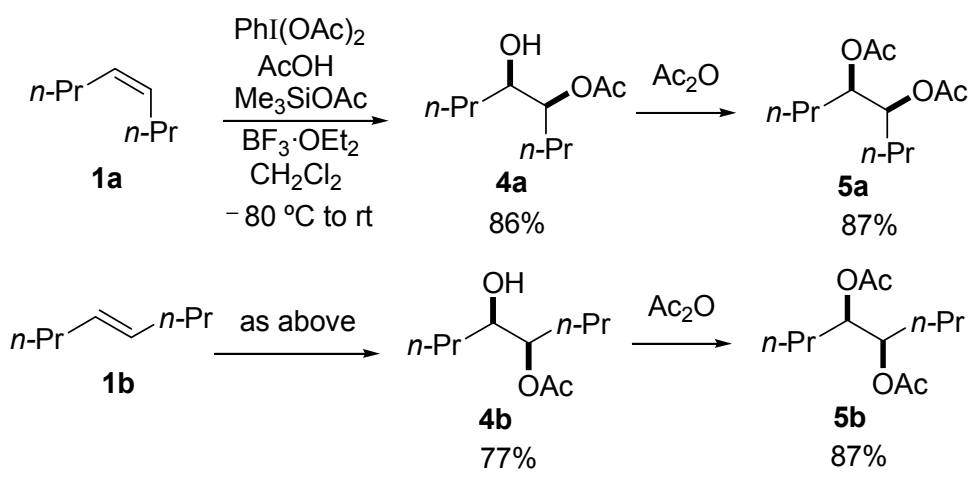

Scheme 2. Simple dioxygenation of 4-octenes $\mathbf{1 a}$ and $\mathbf{1 b}$.

Under optimal conditions (Table 1, entries 3 and 4), the generality of the method was investigated (Table 2). Styrenes and cycloalkenes also successfully gave the dioxolane product $\mathbf{3}$ from reaction with dimethyl ketene silyl acetal 2a (entries 1-6). Isopropenyloxytrimethylsilane (2b), 1-ethoxy-1-trimethylsilyloxyethylene (2c) and 1-phenyl-1-trimethylsilyloxyethylene (2d) were also incorporated into the dioxolane product (entries 7-14) [61]. In the reaction of styrenes, quenching at rt led to a diastereomeric mixture of $\mathbf{3}$ and $\mathbf{3}^{\prime}$ (entries 1 and 3). The diastereoselectivity was highly improved by quenching at a lower temperature (entries 2 and 4). The trans-configuration of the major product 3c or 3d was confirmed by the ${ }^{1} \mathrm{H}-\mathrm{NMR}$ NOESY experiment (SM). The stereochemical outcome may be explained by the steric fence owing to the aryl group during addition of the carbon nucleophile 2a to the 1,3-dioxolan-2-yl cation, similar to the case of the meso cation generated from cis-octene (Scheme 1). Thus, the aryl group at the 4-position of the dioxolanyl cation was able to control the diastereoface selectivity in the nucleophilic trapping. However, the diastereomeric ratio decreased at a higher quenching temperature (entries 1 and 3). To gain an insight into the effect of quenching temperature on diastereoselectivity, the following control experiment was performed. A sample of a single diastereomer of $\mathbf{3 d}$ with trans-configuration was treated with $\mathrm{BF}_{3} \cdot \mathrm{OEt}_{2}$ in a dichloromethane solution at $-40{ }^{\circ} \mathrm{C}$ and then warmed to $\mathrm{rt}$. In the crude reaction mixture, isomerized cis dioxolane 3d' was detected by ${ }^{1} \mathrm{H}-\mathrm{NMR}\left(\mathbf{3 d}(\right.$ trans $) / \mathbf{3} \mathbf{d}^{\prime}($ cis $\left.)=c a .1 / 1\right)$. Thus, the lowering of the diastereomeric ratio may be due to a secondary reaction of the acetal product, i.e., isomerization mediated by a Lewis acid. 
Table 2. Stereoselective formation of dioxolane $3^{\mathrm{a}}$.

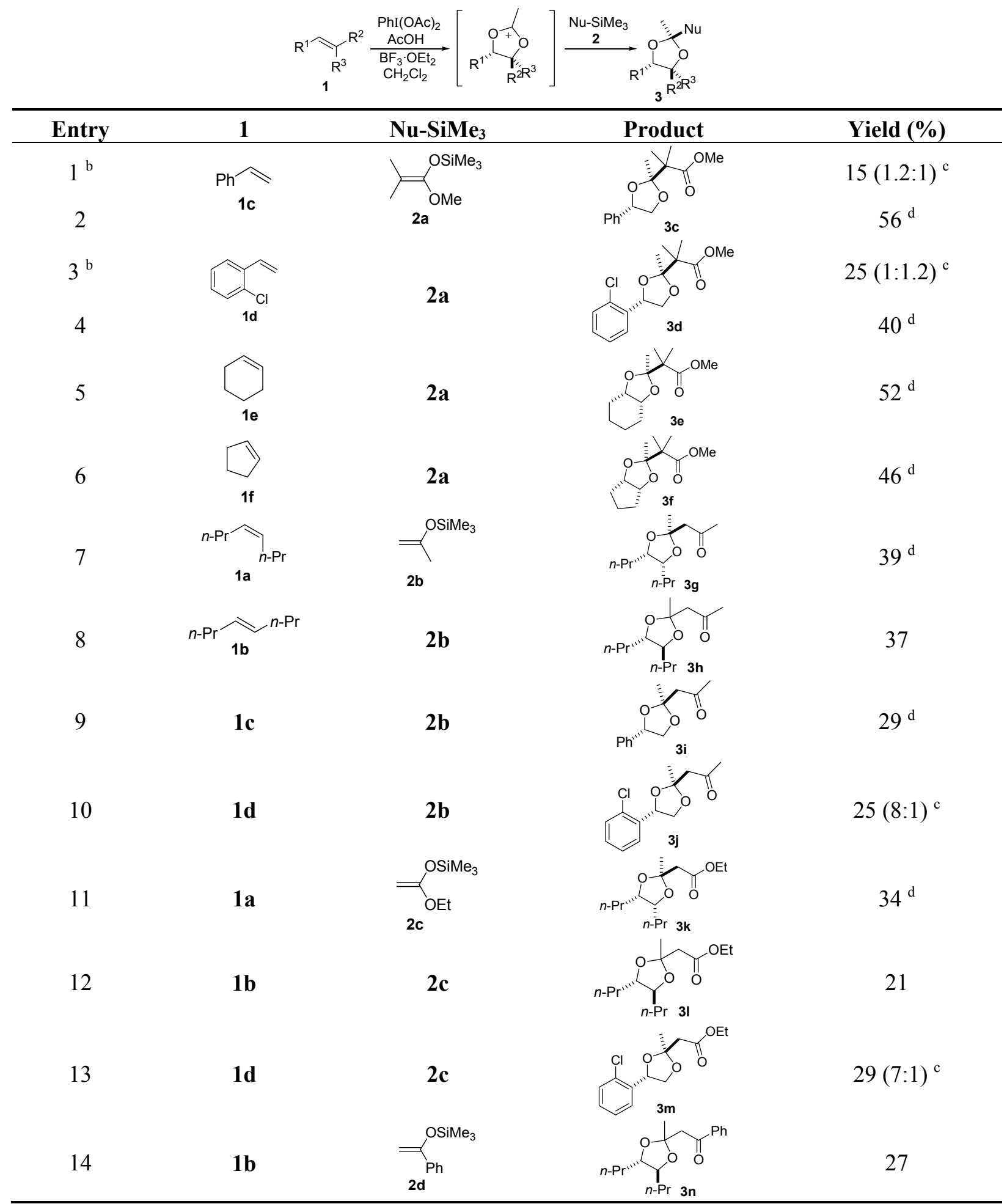

${ }^{\text {a }}$ Reaction was initiated at $-80{ }^{\circ} \mathrm{C}$ in the presence of $1(0.32 \mathrm{mmol}), \mathrm{PhI}(\mathrm{OAc})_{2}(0.40 \mathrm{mmol})$, acetic acid $(0.5 \mathrm{mmol})$ and $\mathrm{BF}_{3} \cdot \mathrm{OEt}_{2}(0.8 \mathrm{mmol})$ in $\mathrm{CH}_{2} \mathrm{Cl}_{2}(4 \mathrm{~mL})$. Then, $2(1.5 \mathrm{mmol})$ was added at $-40{ }^{\circ} \mathrm{C}$ and the reaction mixture was quenched at $-30{ }^{\circ} \mathrm{C}$ by the addition of water; ${ }^{b}$ The reaction was quenched after warming to rt; ${ }^{\mathrm{c}}$ The value in parentheses is the diastereomeric ratio of $\mathbf{3}$ and $\mathbf{3}^{\prime}$ was not detected by ${ }^{1} \mathrm{H}-\mathrm{NMR}$. 
Diastereoselectivity was also affected by the steric effect of the carbon nucleophile; $\beta$-unsubstituted silyl enol ethers $\mathbf{2 b}$ and $\mathbf{2 c}$ led to low diastereoselectivity in the reaction with $\mathbf{1 d}$ (entries 10 and 13, respectively). In contrast, a single diastereomer was formed by the reaction of cis alkene (entries 7 and 11), even with compact $\beta$-unsubstituted nucleophiles. The diastereoselectivity in the reaction of styrenes was affected by the steric effect of the carbon nucleophile 2 . Thus, the stereocontrol by the aryl group in the 4-aryl-1,3-dioxolan-2-yl cation may be slightly reduced compared with the 4,5-dialkyl substituted dioxolanyl cation generated from cis alkene.

Next, a range of carboxylic acid nucleophiles was tested (Table 3). As a primitive examination, (diacetoxyiodo)benzene was used in the reaction of 1d with propanoic acid; however, the protocol resulted in a mixture of $\mathbf{3 d}$ and 3o. Therefore, a set of hypervalent iodine and carboxylic acid compounds was employed as reagents for the reaction. The dioxolane product was obtained from benzoic acid (entry 4), as well as primary and secondary aliphatic carboxylic acids (entries 1 and 2, respectively). NOESY analysis indicated that they have trans-configuration, similar to the acetate-incorporated dioxolane $\mathbf{3 d}$. However, the corresponding dioxolane product was not obtained from the tertiary carboxylic acid (entry 3). In this case, 2-hydroxy-1-arylethyl pivalate 6 was produced as an ordinary oxidation product. The sterically bulky pivalic acid was incorporated into the dioxolane product $3 \mathbf{r}$ when the compact nucleophile 2c was used (Scheme 3). This reaction led to a 1:1 mixture of $\mathbf{3 r}$ and $\mathbf{6}$, and the dioxolane trapping product $\mathbf{3 r}$ was isolated in $10 \%$ yield as a single diastereomer with cis configuration. The relative stereochemistry was confirmed by the nOe observed in the NOESY spectrum (SM), in accordance with stereoface differentiation during nucleophilic addition to the dioxolanyl cation intermediate, as discussed above.

Table 3. Scope of carboxylic acids ${ }^{\text {a }}$.

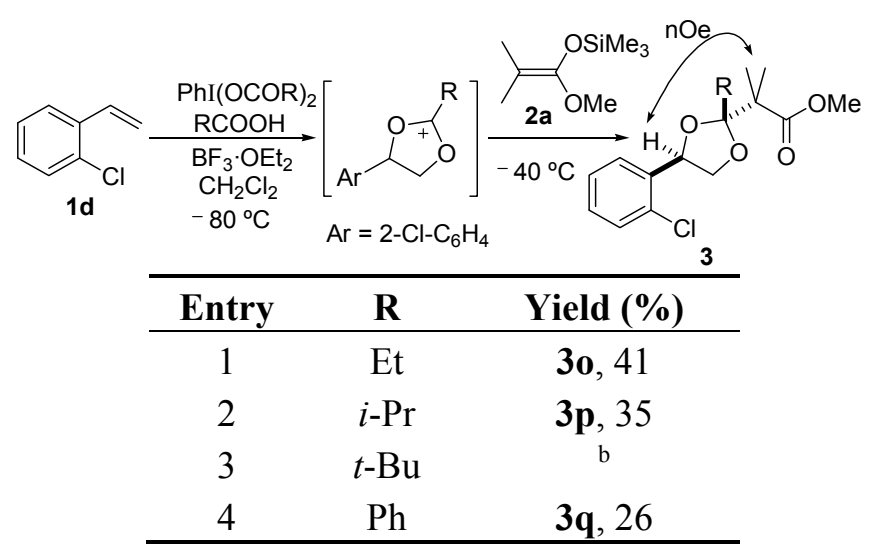

a Reaction was initiated in the presence of $1 \mathbf{d}(0.32 \mathrm{mmol}), \mathrm{PhI}(\mathrm{OCOR})_{2}(0.40 \mathrm{mmol}), \mathrm{RCOOH}(0.5 \mathrm{mmol})$ and $\mathrm{BF}_{3} \cdot \mathrm{OEt}_{2}(0.8 \mathrm{mmol})$ in $\mathrm{CH}_{2} \mathrm{Cl}_{2}(4 \mathrm{~mL})$. Then, $2 \mathbf{a}(1.5 \mathrm{mmol})$ was added at $-40{ }^{\circ} \mathrm{C}$; ${ }^{\mathrm{b}}$ No dioxolane 3 was observed and 2-hydroxy-1-arylethyl pivalate 6 was obtained in 19\% yield.

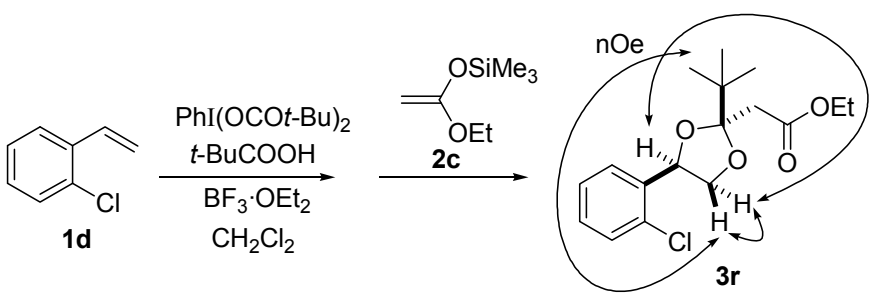

Scheme 3. Reaction with pivalic acid leading to 3r. 
On the basis of the reaction pathway discussed above, we examined enantioselective preparation of the dioxolane product using a chiral hypervalent iodine reagent, as shown in Scheme 4. The lactate-based chiral hypervalent iodine reagent 7 [62-71] has been previously used for enantioselective oxidation of styrenes and has led to high enantioselectivity; a Woodward reaction of $\mathbf{1 d}$ with $\mathbf{7}$ gave the oxyacetylation product with $92 \%$ ee [57]. Comparable enantioselectivity was observed for the three-component assembly but the yield of $\mathbf{3 d}$ was low (20\%). The yield was slightly improved to $36 \%$ when trimethylsilyl acetate was added instead of acetic acid (Scheme 4). An aliphatic alkene 1b was also examined for the enantioselective reaction, and enantioselectivity was slightly decreased in comparison with the styrene substrate. The ee value of the dioxolane $\mathbf{3 h} \mathbf{h}(77 \%$ ee) was comparable with that of the acetoxy product $\mathbf{4 b}(76 \%$ ee) obtained in a separate reaction without addition of the carbon nucleophile $\mathbf{2} \mathbf{b}$.

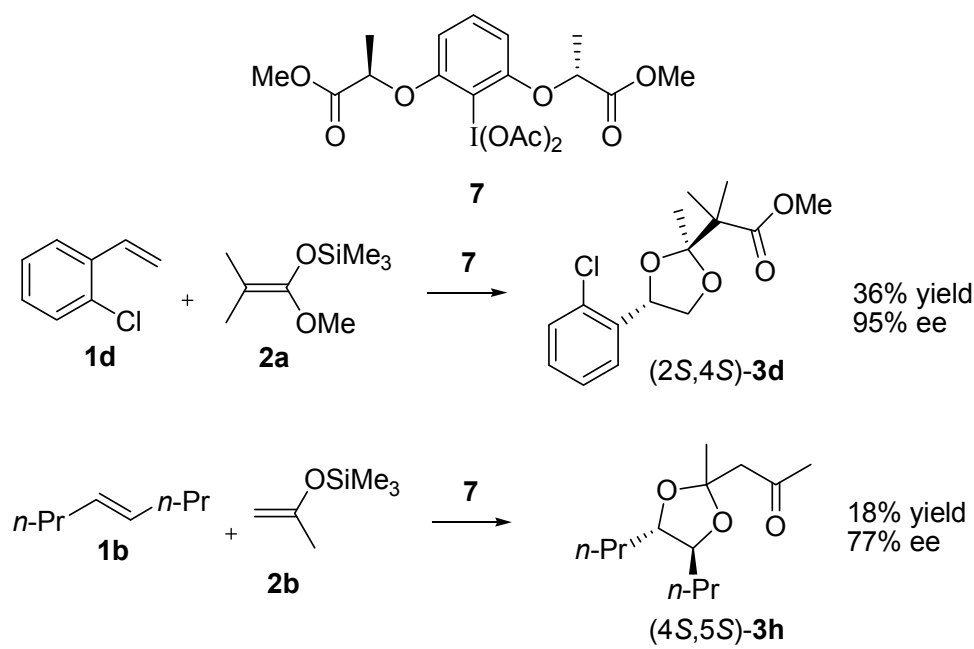

Scheme 4. Enantioselective dioxolane formation.

In conclusion, we achieved the stereoselective formation of 1,3-dioxolane compounds via a 1,3-dioxolan-2-yl cation intermediate, which is stereospecifically generated during the oxidation of alkenes with hypervalent iodine. Three components, alkene, carboxylic acid and carbon nucleophile, were stereoselectively assembled into the dioxolane product over the course of the reaction.

\section{Experimental Section}

\subsection{General Information}

Dichloromethane was purified by distillation over calcium hydride prior to use. Boron trifluoride diethyl etherate was distilled over calcium hydroxide, and kept under nitrogen. All commercial available reagents were used without further purification. (Diacyloxyiodo)benzenes were prepared according to the literature [24]. Reaction temperature was controlled using low temperature baths with magnetic stirrer PSL-1800 (EYELA, Tokyo, Japan) and PSL-1810 (EYELA). Proton and ${ }^{13}$ C-NMR spectra were measured on a JEOL ECA-600 spectrometer (JEOL, Tokyo, Japan) as solutions in $\mathrm{CDCl}_{3}$. Proton NMR spectra were recorded using the residual $\mathrm{CHCl}_{3}$ as an internal reference $(7.24 \mathrm{ppm})$ and ${ }^{13} \mathrm{C}-\mathrm{NMR}$ using $\mathrm{CDCl}_{3}$ as an internal reference $(77.00 \mathrm{ppm})$. For mass spectra measurements was used JEOL JMS-T100LC (JEOL). Optical rotations were measured on a Perkin-Elmer 241 polarimeter (Perkin-Elmer, 
Waltham, MA, USA). Gas chromatography was performed on Shimadzu GC-17A (Shimadzu, Kyoto, Japan) equipped with a chiral column (Chirasil-DEX-CB, $25 \mathrm{~m} \times 0.25 \mathrm{~mm} \times 0.25 \mu \mathrm{m}$ film thickness, Agilent Technology, Santa Clara, CA, USA).

\subsection{Typical Procedure for the Three-Component Assembly Reaction}

(Diacetoxyiodo)benzene (130 mg, $0.40 \mathrm{mmol})$ and cis-4-octene 1a (50 $\mu \mathrm{L}, 0.32 \mathrm{mmol})$ were dissolved in dichloromethane $(4 \mathrm{~mL})$ in the presence of acetic acid $(30 \mu \mathrm{L}, 0.5 \mathrm{mmol})$. The solution was cooled at $-80^{\circ} \mathrm{C}$ using a low temperature bath with magnetic stirrer (EYELA, PSL-1800). Boron trifluoride diethyl etherate $(0.1 \mathrm{~mL}, 0.8 \mathrm{mmol})$ was added to the solution at $-80{ }^{\circ} \mathrm{C}$. The solution was warmed up to $-40{ }^{\circ} \mathrm{C}$ over $1 \mathrm{~h}$. Dimethylketene methyl trimethylsilyl acetal 2a $(0.3 \mathrm{~mL}, 1.5 \mathrm{mmol})$ was added to the solution at $-40{ }^{\circ} \mathrm{C}$ and then the mixture was allowed to warm up to $-30{ }^{\circ} \mathrm{C}$ over $1 \mathrm{~h}$. The reaction mixture was quenched by the addition of water and extracted with dichloromethane. The organic phase was dried with $\mathrm{MgSO}_{4}$ and concentrated in vacuo. The crude mixture was then purified by column chromatography ( $\mathrm{SiO}_{2}$, eluent: $10 \%$ ethyl acetate in hexane) to yield 3a (54.2 mg, $0.20 \mathrm{mmol}$, $62 \%$ yield).

Methyl 2-methyl-2-((2r,4R,5S)-2-methyl-4,5-dipropyl-1,3-dioxolan-2-yl)propanoate (3a). ${ }^{1} \mathrm{H}-\mathrm{NMR}$ $\left(600 \mathrm{MHz} \mathrm{CDCl}_{3}\right) \delta 4.05(\mathrm{~m}, 2 \mathrm{H}), 3.65(\mathrm{~s}, 3 \mathrm{H}), 1.54-1.46(\mathrm{~m}, 4 \mathrm{H}), 1.38(\mathrm{~s}, 3 \mathrm{H}), 1.35-1.27(\mathrm{~m}, 4 \mathrm{H})$, $1.22(\mathrm{~s}, 6 \mathrm{H}), 0.92(\mathrm{t}, J=6.9 \mathrm{~Hz}, 6 \mathrm{H}) ;{ }^{13} \mathrm{C}-\mathrm{NMR}\left(150 \mathrm{MHz}, \mathrm{CDCl}_{3}\right) \delta 176.2,110.8,79.5,52.0,51.9$, 32.4, 25.0, 21.8, 19.6, 14.1; HRMS (ESI-TOF) $\mathrm{m} / \mathrm{z}$ : $[\mathrm{M}+\mathrm{Na}]^{+}$Calcd for $\mathrm{C}_{15} \mathrm{H}_{28} \mathrm{NaO}_{4} 295.1885$; Found 295.1882.

Methyl 2-methyl-2-((4R*,5R*)-2-methyl-4,5-dipropyl-1,3-dioxolan-2-yl)propanoate $\quad(\mathbf{3 b}) . \quad{ }^{1} \mathrm{H}-\mathrm{NMR}$ $\left(600 \mathrm{MHz}, \mathrm{CDCl}_{3}\right) \delta 3.64(\mathrm{~s}, 3 \mathrm{H}), 3.62(\mathrm{~m}, 1 \mathrm{H}), 3.45(\mathrm{~m}, 1 \mathrm{H}), 1.53-1.30(\mathrm{~m}, 8 \mathrm{H}), 1.33(\mathrm{~s}, 3 \mathrm{H}), 1.21$ $(\mathrm{s}, 3 \mathrm{H}), 1.20(\mathrm{~s}, 3 \mathrm{H}), 0.92(\mathrm{t}, J=6.9 \mathrm{~Hz}, 3 \mathrm{H}), 0.91(\mathrm{t}, J=6.9 \mathrm{~Hz}, 3 \mathrm{H}) ;{ }^{13} \mathrm{C}-\mathrm{NMR}\left(150 \mathrm{MHz}, \mathrm{CDCl}_{3}\right)$ $\delta 176.0,110.5,82.1,80.2,51.8,51.1,35.6,34.0,22.3,21.4,21.1,19.3,19.2,14.23,14.16$; HRMS (ESI-TOF) $m / z:[\mathrm{M}+\mathrm{Na}]^{+}$Calcd for $\mathrm{C}_{15} \mathrm{H}_{28} \mathrm{NaO}_{4}$ 295.1885; Found 295.1874.

Methyl 2-methyl-2-((2R*,4R*)-2-methyl-4-phenyl-1,3-dioxolan-2-yl)propanoate (3c). ${ }^{1} \mathrm{H}-\mathrm{NMR}(600 \mathrm{MHz}$, $\left.\mathrm{CDCl}_{3}\right) \delta 7.36-7.26(\mathrm{~m}, 5 \mathrm{H}), 4.96(\mathrm{dd}, J=9.6,6.2 \mathrm{~Hz}, 1 \mathrm{H}), 4.36(\mathrm{dd}, J=8.9,6.2 \mathrm{~Hz}, 1 \mathrm{H}), 3.70(\mathrm{~s}, 3 \mathrm{H})$, $3.67(\mathrm{dd}, J=9.6,8.9 \mathrm{~Hz}, 1 \mathrm{H}), 1.54(\mathrm{~s}, 3 \mathrm{H}), 1.33(\mathrm{~s}, 3 \mathrm{H}), 1.32(\mathrm{~s}, 3 \mathrm{H}) ;{ }^{13} \mathrm{C}-\mathrm{NMR}\left(150 \mathrm{MHz}, \mathrm{CDCl}_{3}\right) \delta$ 175.7, 138.4, 128.6, 128.1, 126.2, 112.8, 79.6, 72.1, 52.0, 51.3, 21.7, 21.6, 20.7; HRMS (ESI-TOF) $m / z:[\mathrm{M}+\mathrm{Na}]^{+}$Calcd for $\mathrm{C}_{15} \mathrm{H}_{20} \mathrm{NaO}_{4}$ 287.1259; Found 287.1256. Selected ${ }^{1} \mathrm{H}-\mathrm{NMR}$ data for the diastereomeric isomer $3 \mathbf{c}^{\prime}:{ }^{1} \mathrm{H}-\mathrm{NMR}\left(600 \mathrm{MHz}, \mathrm{CDCl}_{3}\right) \delta 5.11$ (dd, $\left.J=8.9,6.2 \mathrm{~Hz}, 1 \mathrm{H}\right), 4.28$ (dd, $J=7.6,6.2 \mathrm{~Hz}, 1 \mathrm{H}), 3.69$ (s, 3H), $3.53(\mathrm{dd}, J=8.9,7.6 \mathrm{~Hz}, 1 \mathrm{H})$.

Methyl 2-((2R*,4R*)-4-(2-chlorophenyl)-2-methyl-1,3-dioxolan-2-yl)-2-methylpropanoate (3d). ${ }^{1} \mathrm{H}-\mathrm{NMR}$ $\left(600 \mathrm{MHz}, \mathrm{CDCl}_{3}\right) \delta 7.61(\mathrm{~d}, J=7.6 \mathrm{~Hz}, 1 \mathrm{H}), 7.31-7.26(\mathrm{~m}, 2 \mathrm{H}), 7.21(\mathrm{t}, J=7.6 \mathrm{~Hz}, 1 \mathrm{H}), 5.29$ (dd, $J=9.6$, $6.2 \mathrm{~Hz}, 1 \mathrm{H}), 4.64(\mathrm{dd}, J=8.9,6.2 \mathrm{~Hz}, 1 \mathrm{H}), 3.71(\mathrm{~s}, 3 \mathrm{H}), 3.55(\mathrm{dd}, J=9.6,8.9 \mathrm{~Hz}, 1 \mathrm{H}), 1.51(\mathrm{~s}, 3 \mathrm{H})$, $1.34(\mathrm{~s}, 3 \mathrm{H}), 1.33(\mathrm{~s}, 3 \mathrm{H}) ;{ }^{13} \mathrm{C}-\mathrm{NMR}\left(150 \mathrm{MHz}, \mathrm{CDCl}_{3}\right) \delta 175.7,137.2,131.6,129.2,128.8,127.1$, 126.7, 112.7, 76.5, 70.4, 52.1, 51.2, 21.5, 21.4, 20.9; HRMS (ESI-TOF) $m / z$ : $[\mathrm{M}+\mathrm{Na}]^{+}$Calcd for $\mathrm{C}_{15} \mathrm{H}_{19}{ }^{35} \mathrm{ClNaO}_{4}$ 321.0870; Found 321.0873. Selected ${ }^{1} \mathrm{H}-\mathrm{NMR}$ data for the diastereomeric isomer 3d': 
${ }^{1} \mathrm{H}-\mathrm{NMR}\left(600 \mathrm{MHz}, \mathrm{CDCl}_{3}\right) \delta 7.71(\mathrm{~d}, J=7.6 \mathrm{~Hz}, 1 \mathrm{H}), 5.41(\mathrm{dd}, J=8.9,6.2 \mathrm{~Hz}, 1 \mathrm{H}), 4.55(\mathrm{dd}, J=7.6$, $6.2 \mathrm{~Hz}, 1 \mathrm{H}), 3.69$ (s, 3H), 3.39 (dd, $J=8.9,7.6 \mathrm{~Hz}, 1 \mathrm{H}), 1.48(\mathrm{~s}, 3 \mathrm{H}), 1.35$ (s, 3H), 1.35 (s, 3H).

Methyl 2-((2r,3aS,7aR)-hexahydro-2-methylbenzo[d][1,3]dioxol-2-yl)-2-methylpropanoate (3e). ${ }^{1} \mathrm{H}-\mathrm{NMR}$ $\left(600 \mathrm{MHz}, \mathrm{CDCl}_{3}\right) \delta 4.16(\mathrm{t}, J=4.1 \mathrm{~Hz}, 2 \mathrm{H}), 3.66(\mathrm{~s}, 3 \mathrm{H}), 1.80-1.69(\mathrm{~m}, 4 \mathrm{H}), 1.53-1.47$ (m, 2H), 1.50 (s, $3 \mathrm{H}), 1.25-1.20(\mathrm{~m}, 2 \mathrm{H}), 1.23(\mathrm{~s}, 6 \mathrm{H}) ;{ }^{13} \mathrm{C}-\mathrm{NMR}\left(150 \mathrm{MHz}, \mathrm{CDCl}_{3}\right) \delta 176.2,111.4,75.3,52.4,52.0,28.5$, 24.7, 21.9, 20.5; HRMS (ESI-TOF) $m / z$ : $[\mathrm{M}+\mathrm{Na}]^{+}$Calcd for $\mathrm{C}_{13} \mathrm{H}_{22} \mathrm{NaO}_{4}$ 265.1416; Found 265.1419.

Methyl 2-((2r,3aS,6aR)-tetrahydro-2-methyl-3aH-cyclopenta[d][1,3]dioxol-2-yl)-2-methylpropanoate (3f). ${ }^{1} \mathrm{H}-\mathrm{NMR}\left(600 \mathrm{MHz}, \mathrm{CDCl}_{3}\right) \delta 4.67(\mathrm{t}, J=4.8 \mathrm{~Hz}, 2 \mathrm{H}), 3.66(\mathrm{~s}, 3 \mathrm{H}), 1.86(\mathrm{dd}, J=14.4,6.9 \mathrm{~Hz}$, 2H), $1.70(\mathrm{~m}, 1 \mathrm{H}), 1.55(\mathrm{~m}, 1 \mathrm{H}), 1.41(\mathrm{~s}, 3 \mathrm{H}), 1.39(\mathrm{~m}, 2 \mathrm{H}), 1.21(\mathrm{~s}, 6 \mathrm{H}) ;{ }^{13} \mathrm{C}-\mathrm{NMR}(150 \mathrm{MHz}$, $\left.\mathrm{CDCl}_{3}\right) \delta 176.1,114.5,84.1,52.4,52.0,34.2,23.4,22.6,21.7$; HRMS (ESI-TOF) $\mathrm{m} / z:[\mathrm{M}+\mathrm{Na}]^{+}$ Calcd for $\mathrm{C}_{12} \mathrm{H}_{20} \mathrm{NaO}_{4}$ 251.1259; Found 251.1256.

1-((2r,4S,5R)-2-Methyl-4,5-dipropyl-1,3-dioxolan-2-yl)propan-2-one (3g). ${ }^{1} \mathrm{H}-\mathrm{NMR}\left(600 \mathrm{MHz}, \mathrm{CDCl}_{3}\right)$ $\delta 4.06(\mathrm{~m}, 2 \mathrm{H}), 2.70(\mathrm{~s}, 2 \mathrm{H}), 2.20(\mathrm{~s}, 3 \mathrm{H}), 1.57-1.44(\mathrm{~m}, 4 \mathrm{H}), 1.43(\mathrm{~s}, 3 \mathrm{H}), 1.38-1.26(\mathrm{~m}, 4 \mathrm{H}), 0.93$ $(\mathrm{t}, J=6.9 \mathrm{~Hz}, 6 \mathrm{H}) ;{ }^{13} \mathrm{C}-\mathrm{NMR}\left(150 \mathrm{MHz}, \mathrm{CDCl}_{3}\right) \delta 206.6,106.4,78.2,53.1,31.8,31.5,27.0,19.4$, 14.1; HRMS (ESI-TOF) $m / z$ : [M + Na $]^{+}$Calcd for $\mathrm{C}_{13} \mathrm{H}_{24} \mathrm{NaO}_{3} 251.1623$; Found 251.1619.

1-((4R*,5R*)-2-Methyl-4,5-dipropyl-1,3-dioxolan-2-yl)propan-2-one (3h). ${ }^{1} \mathrm{H}-\mathrm{NMR}\left(600 \mathrm{MHz}, \mathrm{CDCl}_{3}\right)$ $\delta 3.64-3.53(\mathrm{~m}, 2 \mathrm{H}), 2.72(\mathrm{~s}, 2 \mathrm{H}), 2.20(\mathrm{~s}, 3 \mathrm{H}), 1.52-1.43(\mathrm{~m}, 6 \mathrm{H}), 1.42-1.30(\mathrm{~m}, 2 \mathrm{H}), 1.37(\mathrm{~s}, 3 \mathrm{H})$, $0.92(\mathrm{t}, J=6.9 \mathrm{~Hz}, 6 \mathrm{H}) ;{ }^{13} \mathrm{C}-\mathrm{NMR}\left(150 \mathrm{MHz}, \mathrm{CDCl}_{3}\right) \delta 206.5,107.0,81.2,80.8,54.1,34.9,34.7,31.8$, 26.1 19.40, 19.37, 14.2, 14.1; HRMS (ESI-TOF) $m / z$ : $[\mathrm{M}+\mathrm{Na}]^{+}$Calcd for $\mathrm{C}_{13} \mathrm{H}_{24} \mathrm{NaO}_{3} 251.1623$; Found 251.1626.

1-((2R*,4R*)-2-Methyl-4-phenyl-1,3-dioxolan-2-yl)propan-2-one (3i). ${ }^{1} \mathrm{H}-\mathrm{NMR}\left(600 \mathrm{MHz}, \mathrm{CDCl}_{3}\right)$ $\delta 7.38-7.27(\mathrm{~m}, 5 \mathrm{H}), 5.07(\mathrm{dd}, J=8.9,6.2 \mathrm{~Hz}, 1 \mathrm{H}), 4.33(\mathrm{dd}, J=8.9,6.2 \mathrm{~Hz}, 1 \mathrm{H}), 3.72(\mathrm{t}, J=8.9 \mathrm{~Hz}$, 1H), 2.87 (s, 2H), 2.25 (s, 3H), $1.57(\mathrm{~s}, 3 \mathrm{H}) ;{ }^{13} \mathrm{C}-\mathrm{NMR}\left(150 \mathrm{MHz}, \mathrm{CDCl}_{3}\right) \delta 205.9,138.3,128.6,128.2$, 126.2, 108.9, 78.4, 71.7, 52.8, 31.8, 25.3; HRMS (ESI-TOF) $m / z:[\mathrm{M}+\mathrm{Na}]^{+}$Calcd for $\mathrm{C}_{13} \mathrm{H}_{16} \mathrm{NaO}_{3}$ 243.0997; Found 243.0999.

1-((2R*,4R*)-4-(2-Chlorophenyl)-2-methyl-1,3-dioxolan-2-yl)propan-2-one (3j). ${ }^{1} \mathrm{H}-\mathrm{NMR}(600 \mathrm{MHz}$, $\left.\mathrm{CDCl}_{3}\right) \delta 7.60(\mathrm{~d}, J=7.6 \mathrm{~Hz}, 1 \mathrm{H}), 7.32(\mathrm{~d}, J=7.6 \mathrm{~Hz}, 1 \mathrm{H}), 7.29(\mathrm{t}, J=7.6 \mathrm{~Hz}, 1 \mathrm{H}), 7.22(\mathrm{t}, J=7.6 \mathrm{~Hz}$, $1 \mathrm{H}), 5.41(\mathrm{dd}, J=7.6,6.9 \mathrm{~Hz}, 1 \mathrm{H}), 4.58(\mathrm{dd}, J=8.2,6.9 \mathrm{~Hz}, 1 \mathrm{H}), 3.65(\mathrm{dd}, J=8.2,7.6 \mathrm{~Hz}, 1 \mathrm{H}), 2.89$ (s, 2H), $2.25(\mathrm{~s}, 3 \mathrm{H}), 1.58(\mathrm{~s}, 3 \mathrm{H}) ;{ }^{13} \mathrm{C}-\mathrm{NMR}\left(150 \mathrm{MHz}, \mathrm{CDCl}_{3}\right) \delta 205.8,137.2,131.6,129.2,128.9$, 127.1, 126.6, 108.9, 75.3, 70.3, 52.5, 31.7, 24.9; HRMS (ESI-TOF) $m / z$ : $\left[\mathrm{M}+\mathrm{Na}^{+}\right.$Calcd for $\mathrm{C}_{13} \mathrm{H}_{15}{ }^{35} \mathrm{ClNaO}_{3}$ 277.0607; Found 277.0613. Selected ${ }^{1} \mathrm{H}-\mathrm{NMR}$ data for the diastereomeric isomer $\mathbf{3 j}$ ': ${ }^{1} \mathrm{H}-\mathrm{NMR}\left(600 \mathrm{MHz}, \mathrm{CDCl}_{3}\right) \delta 7.58(\mathrm{~d}, J=7.6 \mathrm{~Hz}, 1 \mathrm{H}), 4.56(\mathrm{dd}, J=8.2,6.9 \mathrm{~Hz}, 1 \mathrm{H}), 3.61$ (dd, $J=8.2$, $7.6 \mathrm{~Hz}, 1 \mathrm{H}), 2.93$ (s, 2H), 2.25 (s, 3H), 1.53 (s, 3H).

Ethyl 2-((2r,4S,5R)-2-methyl-4,5-dipropyl-1,3-dioxolan-2-yl)acetate (3k). ${ }^{1} \mathrm{H}-\mathrm{NMR}\left(600 \mathrm{MHz}, \mathrm{CDCl}_{3}\right) \delta$ $4.11(\mathrm{q}, J=6.9 \mathrm{~Hz}, 2 \mathrm{H}), 4.06(\mathrm{~m}, 2 \mathrm{H}), 2.62(\mathrm{~s}, 2 \mathrm{H}), 1.53(\mathrm{~s}, 3 \mathrm{H}), 1.52-1.40(\mathrm{~m}, 4 \mathrm{H}), 1.37-1.25(\mathrm{~m}, 4 \mathrm{H})$, $1.23(\mathrm{t}, J=6.9 \mathrm{~Hz}, 3 \mathrm{H}), 0.92(\mathrm{t}, J=6.9 \mathrm{~Hz}, 6 \mathrm{H}) ;{ }^{13} \mathrm{C}-\mathrm{NMR}\left(150 \mathrm{MHz}, \mathrm{CDCl}_{3}\right) \delta 169.7,106.3,78.1$, 
60.4, 44.6, 31.8, 27.0, 19.4, 14.2, 14.1; HRMS (ESI-TOF) $m / z:[\mathrm{M}+\mathrm{Na}]^{+}$Calcd for $\mathrm{C}_{14} \mathrm{H}_{26} \mathrm{NaO}_{4}$ 281.1729; Found 281.1716.

Ethyl 2-((4R*,5R*)-2-methyl-4,5-dipropyl-1,3-dioxolan-2-yl)acetate (31). ${ }^{1} \mathrm{H}-\mathrm{NMR}\left(600 \mathrm{MHz}, \mathrm{CDCl}_{3}\right)$ $\delta 4.12(\mathrm{q}, J=6.9 \mathrm{~Hz}, 2 \mathrm{H}), 3.64(\mathrm{td}, J=8.2,4.1 \mathrm{~Hz}, 1 \mathrm{H}), 3.60(\mathrm{td}, J=8.2,4.1 \mathrm{~Hz}, 1 \mathrm{H}), 2.64$ (d, $J=13.1 \mathrm{~Hz}$, $1 \mathrm{H}), 2.62(\mathrm{~d}, J=13.1 \mathrm{~Hz}, 1 \mathrm{H}), 1.53-1.42(\mathrm{~m}, 6 \mathrm{H}), 1.48(\mathrm{~s}, 3 \mathrm{H}), 1.41-1.32(\mathrm{~m}, 2 \mathrm{H}), 1.24(\mathrm{t}, J=6.9 \mathrm{~Hz}$, $3 \mathrm{H}), 0.92(\mathrm{t}, J=6.9 \mathrm{~Hz}, 6 \mathrm{H}) ;{ }^{13} \mathrm{C}-\mathrm{NMR}\left(150 \mathrm{MHz}, \mathrm{CDCl}_{3}\right) \delta 169.6,106.8,81.1,81.0,60.4,45.7,34.8$, $34.7,26.2,19.30,19.26,14.19,14.17,14.1$; HRMS (ESI-TOF) $m / z:[\mathrm{M}+\mathrm{Na}]^{+}$Calcd for $\mathrm{C}_{14} \mathrm{H}_{26} \mathrm{NaO}_{4}$ 281.1729; Found 281.1721.

Ethyl 2-((2R*,4R*)-4-(2-chlorophenyl)-2-methyl-1,3-dioxolan-2-yl)acetate (3m). ${ }^{1} \mathrm{H}-\mathrm{NMR}(600 \mathrm{MHz}$, $\left.\mathrm{CDCl}_{3}\right) \delta 7.60(\mathrm{~d}, J=7.6 \mathrm{~Hz}, 1 \mathrm{H}), 7.31(\mathrm{~d}, J=7.6 \mathrm{~Hz}, 1 \mathrm{H}), 7.28(\mathrm{t}, J=7.6 \mathrm{~Hz}, 1 \mathrm{H}), 7.21(\mathrm{t}, J=7.6 \mathrm{~Hz}$, $1 \mathrm{H}), 5.45(\mathrm{dd}, J=7.6,6.9 \mathrm{~Hz}, 1 \mathrm{H}), 4.57(\mathrm{dd}, J=8.2,6.9 \mathrm{~Hz}, 1 \mathrm{H}), 4.17$ (q, $J=6.9 \mathrm{~Hz}, 2 \mathrm{H}), 3.66(\mathrm{dd}$, $J=8.2,7.6 \mathrm{~Hz}, 1 \mathrm{H}), 2.79(\mathrm{~s}, 2 \mathrm{H}), 1.68(\mathrm{~s}, 3 \mathrm{H}), 1.27(\mathrm{t}, J=6.9 \mathrm{~Hz}, 3 \mathrm{H}) ;{ }^{13} \mathrm{C}-\mathrm{NMR}\left(150 \mathrm{MHz}, \mathrm{CDCl}_{3}\right)$ $\delta 169.3,137.4,131.6,129.2,128.8,127.0,126.7,108.7,75.3,70.6,60.7,44.2,25.0,14.2$; HRMS (ESI-TOF) $m / z$ : $[\mathrm{M}+\mathrm{Na}]^{+}$Calcd for $\mathrm{C}_{14} \mathrm{H}_{17}{ }^{35} \mathrm{ClNaO}_{4}$ 307.0713; Found 307.0711. Selected ${ }^{1} \mathrm{H}-\mathrm{NMR}$ data for the diastereomeric isomer 3m': ${ }^{1} \mathrm{H}-\mathrm{NMR}\left(600 \mathrm{MHz}, \mathrm{CDCl}_{3}\right) \delta 7.69(\mathrm{~d}, J=7.6 \mathrm{~Hz}, 1 \mathrm{H}), 5.42$ $(\mathrm{dd}, J=8.2,6.9 \mathrm{~Hz}, 1 \mathrm{H}), 4.17$ (q, $J=6.9 \mathrm{~Hz}, 2 \mathrm{H}), 3.59$ (t, $J=8.2 \mathrm{~Hz}, 1 \mathrm{H}), 2.82(\mathrm{~s}, 2 \mathrm{H}), 1.62$ (s, 3H).

2-((4R*,5R*)-2-Methyl-4,5-dipropyl-1,3-dioxolan-2-yl)-1-phenylethanone (3n). ${ }^{1} \mathrm{H}-\mathrm{NMR}$ (600 MHz, $\left.\mathrm{CDCl}_{3}\right) \delta 7.97(\mathrm{~d}, J=7.6 \mathrm{~Hz}, 2 \mathrm{H}), 7.52(\mathrm{t}, J=7.6 \mathrm{~Hz}, 1 \mathrm{H}), 7.42(\mathrm{t}, J=7.6 \mathrm{~Hz}, 2 \mathrm{H}), 3.56(\mathrm{td}, J=7.6,3.4$ $\mathrm{Hz}, 1 \mathrm{H}), 3.42(\mathrm{td}, J=7.6,3.4 \mathrm{~Hz}, 1 \mathrm{H}), 3.30$ (d, $J=13.7 \mathrm{~Hz}, 1 \mathrm{H}), 3.27$ (d, $J=13.7 \mathrm{~Hz}, 1 \mathrm{H}), 1.48$ (s, $3 \mathrm{H}), 1.45-1.18(\mathrm{~m}, 8 \mathrm{H}), 0.85(\mathrm{t}, J=6.9 \mathrm{~Hz}, 3 \mathrm{H}), 0.84(\mathrm{t}, J=6.9 \mathrm{~Hz}, 3 \mathrm{H}) ;{ }^{13} \mathrm{C}-\mathrm{NMR}(150 \mathrm{MHz}$, $\left.\mathrm{CDCl}_{3}\right) \delta 197.6,138.0,132.8,129.0,128.2,107.6,81.2,80.6,48.9,34.6,34.3,26.8,19.3,19.2$, 14.11, 14.08; HRMS (ESI-TOF) $m / z$ : $[\mathrm{M}+\mathrm{Na}]^{+}$Calcd for $\mathrm{C}_{18} \mathrm{H}_{26} \mathrm{NaO}_{3} 313.1780$; Found 313.1777.

Methyl 2-((2R*,4R*)-4-(2-chlorophenyl)-2-ethyl-1,3-dioxolan-2-yl)-2-methylpropanoate (3o). ${ }^{1} \mathrm{H}-\mathrm{NMR}$ $\left(600 \mathrm{MHz}, \mathrm{CDCl}_{3}\right) \delta 7.73(\mathrm{~d}, J=7.6 \mathrm{~Hz}, 1 \mathrm{H}), 7.31(\mathrm{~d}, J=7.6 \mathrm{~Hz}, 1 \mathrm{H}), 7.29(\mathrm{t}, J=7.6 \mathrm{~Hz}, 1 \mathrm{H}), 7.21$ (t, $J=7.6 \mathrm{~Hz}, 1 \mathrm{H}), 5.37(\mathrm{dd}, J=9.6,6.9 \mathrm{~Hz}, 1 \mathrm{H}), 4.70(\mathrm{dd}, J=8.2,6.9 \mathrm{~Hz}, 1 \mathrm{H}), 3.70(\mathrm{~s}, 3 \mathrm{H}), 3.58$ (dd, $J=9.6,8.2 \mathrm{~Hz}, 1 \mathrm{H}), 1.96(\mathrm{dq}, J=15.1,7.6 \mathrm{~Hz}, 1 \mathrm{H}), 1.94(\mathrm{dq}, J=15.1,7.6 \mathrm{~Hz}, 1 \mathrm{H}), 1.33(\mathrm{~s}, 3 \mathrm{H}), 1.32$ $(\mathrm{s}, 3 \mathrm{H}), 0.92(\mathrm{t}, J=7.6 \mathrm{~Hz}, 3 \mathrm{H}) ;{ }^{13} \mathrm{C}-\mathrm{NMR}\left(150 \mathrm{MHz}, \mathrm{CDCl}_{3}\right) \delta 176.0,136.8,131.5,129.2,128.7$, 127.0, 126.4, 114.5, 76.5, 72.1, 52.2, 52.1, 28.4, 21.9, 21.6, 8.1; HRMS (ESI-TOF) $m / z:[\mathrm{M}+\mathrm{Na}]^{+}$ Calcd for $\mathrm{C}_{16} \mathrm{H}_{21}{ }^{35} \mathrm{ClNaO}_{4} 335.1026$; Found 335.1014.

Methyl 2-((2R*,4R*)-4-(2-chlorophenyl)-2-isopropyl-1,3-dioxolan-2-yl)-2-methylpropanoate (3p). ${ }^{1} \mathrm{H}-\mathrm{NMR}\left(600 \mathrm{MHz}, \mathrm{CDCl}_{3}\right) \delta 7.75(\mathrm{~d}, J=7.6 \mathrm{~Hz}, 1 \mathrm{H}), 7.30(\mathrm{t}, J=7.6 \mathrm{~Hz}, 1 \mathrm{H}), 7.28(\mathrm{~d}, J=7.6 \mathrm{~Hz}$, 1H), $7.21(\mathrm{t}, J=7.6 \mathrm{~Hz}, 1 \mathrm{H}), 5.44(\mathrm{dd}, J=9.6,6.9 \mathrm{~Hz}, 1 \mathrm{H}), 4.70(\mathrm{t}, J=6.9 \mathrm{~Hz}, 1 \mathrm{H}), 3.69$ (s, 3H), 3.53 (dd, $J=9.6,6.9 \mathrm{~Hz}, 1 \mathrm{H}), 2.38$ (sept, $J=6.9 \mathrm{~Hz}, 1 \mathrm{H}), 1.36(\mathrm{~s}, 3 \mathrm{H}), 1.34$ (s, 3H), 1.04 (d, $J=6.9 \mathrm{~Hz}$, $3 \mathrm{H}), 0.94(\mathrm{~d}, J=6.9 \mathrm{~Hz}, 3 \mathrm{H}) ;{ }^{13} \mathrm{C}-\mathrm{NMR}\left(150 \mathrm{MHz}, \mathrm{CDCl}_{3}\right) \delta 176.3,137.0,131.4,129.3,128.7,127.0$, 126.5, 115.2, 76.7, 71.6, 52.4, 52.0, 34.9, 22.6, 22.5, 19.1, 18.3; HRMS (ESI-TOF) $\mathrm{m} / z$ : $[\mathrm{M}+\mathrm{Na}]^{+}$ Calcd for $\mathrm{C}_{17} \mathrm{H}_{23}{ }^{35} \mathrm{ClNaO}_{4} 349.1183$; Found 349.1186. 
Methyl 2-((2R*,4R*)-4-(2-chlorophenyl)-2-phenyl-1,3-dioxolan-2-yl)-2-methylpropanoate (3q). ${ }^{1} \mathrm{H}-\mathrm{NMR}$ $\left(600 \mathrm{MHz}, \mathrm{CDCl}_{3}\right) \delta 7.54(\mathrm{~d}, J=7.6 \mathrm{~Hz}, 2 \mathrm{H}), 7.41(\mathrm{t}, J=7.6 \mathrm{~Hz}, 1 \mathrm{H}), 7.35(\mathrm{t}, J=7.6 \mathrm{~Hz}, 2 \mathrm{H}), 7.30$ $(\mathrm{t}, J=7.6 \mathrm{~Hz}, 1 \mathrm{H}), 7.25(\mathrm{t}, J=7.6 \mathrm{~Hz}, 1 \mathrm{H}), 7.14(\mathrm{~d}, J=7.6 \mathrm{~Hz}, 1 \mathrm{H}), 7.13(\mathrm{~d}, J=7.6 \mathrm{~Hz}, 1 \mathrm{H}), 5.46(\mathrm{dd}$, $J=9.6,5.5 \mathrm{~Hz}, 1 \mathrm{H}), 4.61(\mathrm{dd}, J=8.2,5.5 \mathrm{~Hz}, 1 \mathrm{H}), 3.65(\mathrm{~s}, 3 \mathrm{H}), 3.24(\mathrm{dd}, J=9.6,8.2 \mathrm{~Hz}, 1 \mathrm{H}), 1.33(\mathrm{~s}, 3 \mathrm{H})$, $1.27(\mathrm{~s}, 3 \mathrm{H}) ;{ }^{13} \mathrm{C}-\mathrm{NMR}\left(150 \mathrm{MHz}, \mathrm{CDCl}_{3}\right) \delta 175.0,140.1,135.9,131.9,129.1,128.8,128.2,127.7$, 127.5, 127.3, 127.0, 112.4, 77.6, 70.6, 51.9, 51.5, 21.5, 21.2; HRMS (ESI-TOF) $\mathrm{m} / \mathrm{z}:[\mathrm{M}+\mathrm{Na}]^{+} \mathrm{Calcd}$ for $\mathrm{C}_{20} \mathrm{H}_{21}{ }^{35} \mathrm{ClNaO}_{4}$ 383.1026; Found 383.1013.

Ethyl 2-((2R*,4S*)-2-tert-butyl-4-(2-chlorophenyl)-1,3-dioxolan-2-yl)acetate (3r). ${ }^{1} \mathrm{H}-\mathrm{NMR}(600 \mathrm{MHz}$, $\left.\mathrm{CDCl}_{3}\right) \delta 7.70(\mathrm{~d}, J=7.6 \mathrm{~Hz}, 1 \mathrm{H}), 7.31(\mathrm{~d}, J=7.6 \mathrm{~Hz}, 1 \mathrm{H}), 7.28(\mathrm{t}, J=7.6 \mathrm{~Hz}, 1 \mathrm{H}), 7.21(\mathrm{t}, J=7.6 \mathrm{~Hz}$, $1 \mathrm{H}), 5.52(\mathrm{dd}, J=9.6,6.9 \mathrm{~Hz}, 1 \mathrm{H}), 4.66(\mathrm{t}, J=6.9 \mathrm{~Hz}, 1 \mathrm{H}), 4.19(\mathrm{dq}, J=9.6,6.9 \mathrm{~Hz}, 1 \mathrm{H}), 4.16(\mathrm{dq}, J=9.6$, $6.9 \mathrm{~Hz}, 1 \mathrm{H}), 3.47(\mathrm{dd}, J=9.6,6.9 \mathrm{~Hz}, 1 \mathrm{H}), 2.88(\mathrm{~d}, J=13.7 \mathrm{~Hz}, 1 \mathrm{H}), 2.82(\mathrm{~d}, J=13.7 \mathrm{~Hz}, 1 \mathrm{H}), 1.29$ $(\mathrm{t}, J=6.9 \mathrm{~Hz}, 3 \mathrm{H}), 1.06(\mathrm{~s}, 9 \mathrm{H}) ;{ }^{13} \mathrm{C}-\mathrm{NMR}\left(150 \mathrm{MHz}, \mathrm{CDCl}_{3}\right) \delta 170.7,136.6,131.5,129.3,128.7$, 127.0, 126.4, 114.2, 75.8, 71.6, 60.7, 40.8, 40.6, 25.4, 14.2; HRMS (ESI-TOF) $m / z:[\mathrm{M}+\mathrm{Na}]^{+} \mathrm{Calcd}$ for $\mathrm{C}_{17} \mathrm{H}_{23}{ }^{35} \mathrm{ClNaO}_{4} 349.1183$; Found 349.1182.

1-(2-Chlorophenyl)-2-hydroxyethyl pivalate (6). ${ }^{1} \mathrm{H}-\mathrm{NMR}\left(600 \mathrm{MHz}, \mathrm{CDCl}_{3}\right) \delta 7.59(\mathrm{~d}, J=7.6 \mathrm{~Hz}$, $1 \mathrm{H}), 7.33(\mathrm{~d}, J=7.6 \mathrm{~Hz}, 1 \mathrm{H}), 7.29(\mathrm{t}, J=7.6 \mathrm{~Hz}, 1 \mathrm{H}), 7.23(\mathrm{t}, J=7.6 \mathrm{~Hz}, 1 \mathrm{H}), 5.36$ (dd, $J=7.6$, $2.7 \mathrm{~Hz}, 1 \mathrm{H}), 4.35$ (dd, $J=11.7,2.7 \mathrm{~Hz}, 1 \mathrm{H}), 4.20$ (dd, $J=11.7,7.6 \mathrm{~Hz}, 1 \mathrm{H}), 1.18(\mathrm{~s}, 9 \mathrm{H}) ;{ }^{13} \mathrm{C}-\mathrm{NMR}$ $\left(150 \mathrm{MHz}, \mathrm{CDCl}_{3}\right) \delta 179.1,137.3,132.0,129.4,129.1,127.8,127.0,69.6,67.8,38.9,27.2$; HRMS (ESI-TOF) $m / z:[\mathrm{M}+\mathrm{Na}]^{+}$Calcd for $\mathrm{C}_{13} \mathrm{H}_{17}{ }^{35} \mathrm{ClNaO}_{3} 279.0764$; Found 279.0761.

\subsection{Isomerization of $\mathbf{3 d}$ Mediated by a Lewis Acid}

Boron trifluoride diethyl etherate $(0.1 \mathrm{~mL}, 0.8 \mathrm{mmol})$ was added to a dichloromethane solution $(4 \mathrm{~mL})$ containing $3 \mathbf{d}$ ( $36.2 \mathrm{mg}, 0.12 \mathrm{mmol})$ at $-40{ }^{\circ} \mathrm{C}$. The solution was gradually warmed up to $0{ }^{\circ} \mathrm{C}$ over $1 \mathrm{~h}$. The reaction mixture was quenched by the addition of water and extracted with dichloromethane. The organic phase was dried with $\mathrm{Na}_{2} \mathrm{SO}_{4}$ and concentrated in vacuo. The crude mixture contained 3d and the diastereomer $\mathbf{3} \mathbf{d}^{\prime}$ in a 45 to 55 ratio, in addition to decomposed products. The crude mixture was purified by column chromatography ( $\mathrm{SiO}_{2}$, eluent: $10 \%$ ethyl acetate in hexane) to give a diastereomeric mixture (3d/3 $\mathbf{d}^{\prime}=48 / 52,8.4 \mathrm{mg}, 23 \%$ yield $)$.

\subsection{Enantioselective Reaction of $\mathbf{1} \mathbf{d}$ with $\mathbf{7}$ and $\mathbf{2 a}$}

The lactate-based hypervalent iodine(III) reagent $7(260 \mathrm{mg}, 0.50 \mathrm{mmol})$ and 2-chlorostyrene 1d $(55 \mu \mathrm{L}, 0.43 \mathrm{mmol})$ were dissolved in dichloromethane $(4 \mathrm{~mL})$ in the presence of trimethylsilyl acetate $(30 \mu \mathrm{L}, 0.20 \mathrm{mmol})$. The solution was cooled at $-80{ }^{\circ} \mathrm{C}$ using a low temperature bath with magnetic stirrer (EYELA, PSL-1800). Boron trifluoride diethyl etherate $(0.1 \mathrm{~mL}, 0.8 \mathrm{mmol})$ was added to the solution at $-80^{\circ} \mathrm{C}$ and the mixture was stirred for $1 \mathrm{~h}$ at $-80^{\circ} \mathrm{C}$. Dimethylketene methyl trimethylsilyl acetal $2 \mathrm{a}(0.3 \mathrm{~mL}, 1.5 \mathrm{mmol})$ was added to the solution at $-80{ }^{\circ} \mathrm{C}$ and the mixture was stirred for $30 \mathrm{~min}$ at $-80{ }^{\circ} \mathrm{C}$. The reaction mixture was quenched by the addition of water and extracted with dichloromethane. The organic phase was dried with $\mathrm{Na}_{2} \mathrm{SO}_{4}$ and concentrated in vacuo. The crude mixture was purified by column chromatography ( $\mathrm{SiO}_{2}$, eluent: $10 \%$ ethyl acetate in hexane) to give $\mathbf{3 d}$ (46.6 $\mathrm{mg}, 0.16 \mathrm{mmol}$, 
$36 \%$ yield). Selected data for $(2 S, 4 S)-3 \mathbf{d}$ : $[\alpha]_{\mathrm{D}}^{20}+33.5\left(c 0.83\right.$ in $\left.\mathrm{CH}_{2} \mathrm{Cl}_{2}\right)$. Ee of $\mathbf{3 d}$ was determined to be $95 \%$ by GC on a chiral stationary phase (DEX-CB, i.d. $0.25 \mathrm{~mm} \times 25 \mathrm{~m})$. Retention times of $(2 R, 4 R)-3 \mathbf{d}$ and $(2 S, 4 S)-3 \mathbf{d}$ were 30.1 and $31.1 \mathrm{~min}$, respectively, when the column temperature was maintained at $160{ }^{\circ} \mathrm{C}$. The absolute stereochemistry was based on the analogy of the enantioselective Woodward reaction of $\mathbf{1 d}$ [57].

\subsection{Enantioselective Reaction of $\mathbf{1 b}$ with $\mathbf{7}$ and $\mathbf{2 b}$}

Boron trifluoride diethyl etherate $(0.1 \mathrm{~mL}, 0.8 \mathrm{mmol})$ was added to a dichloromethane solution $(4 \mathrm{~mL})$ containing 7 (260 $\mathrm{mg}, 0.50 \mathrm{mmol}), \mathbf{1 b}(68 \mu \mathrm{L}, 0.43 \mathrm{mmol})$ and trimethylsilyl acetate $(30 \mu \mathrm{L}, 0.20 \mathrm{mmol})$ at $-80{ }^{\circ} \mathrm{C}$. The solution was warmed to $-40{ }^{\circ} \mathrm{C}$ over $1 \mathrm{~h}$ and $\mathbf{2 b}(0.3 \mathrm{~mL}, 1.8 \mathrm{mmol})$ was subsequently added to the solution at $-40{ }^{\circ} \mathrm{C}$. The mixture was warmed to $-30{ }^{\circ} \mathrm{C}$ over $1 \mathrm{~h}$ and quenched by the addition of water. Extracts with dichloromethane were dried with $\mathrm{Na}_{2} \mathrm{SO}_{4}$ and concentrated in vacuo. The crude mixture was purified by column chromatography $\left(\mathrm{SiO}_{2}\right.$, eluent: $10 \%$ ethyl acetate in hexane) to give $\mathbf{3 h}$ (17.9 mg, $0.078 \mathrm{mmol}, 18 \%$ yield). Selected data for $(4 S, 5 S)-3 \mathbf{h}:[\alpha]_{\mathrm{D}}^{20}-18.9(c 0.36$ in $\mathrm{CH}_{2} \mathrm{Cl}_{2}$ ). Ee of $\mathbf{3 h}$ was determined to be $77 \%$ by GC on a chiral stationary phase (DEX-CB, i.d. $0.25 \mathrm{~mm} \times 25 \mathrm{~m})$. Retention times of $(4 R, 5 R)-\mathbf{3 h}$ and $(4 S, 5 S)$-3h were 39.6 and $40.2 \mathrm{~min}$, respectively, when the column temperature was maintained at $100{ }^{\circ} \mathrm{C}$. The absolute stereochemistry was based on the analogy of the enantioselective Woodward reaction of $\mathbf{1 b}$, which was described below.

$\left(4 R^{*}, 5 S^{*}\right)-5$-Hydroxyoctan-4-yl acetate $4 \mathbf{a}$ : Boron trifluoride diethyl etherate $(0.1 \mathrm{~mL}, 0.8 \mathrm{mmol})$ was added to a dichloromethane solution $(4 \mathrm{~mL})$ containing $\mathrm{PhI}(\mathrm{OAc})_{2}(130 \mathrm{mg}, 0.40 \mathrm{mmol}), \mathbf{1 a}$ $(50 \mu \mathrm{L}, 0.32 \mathrm{mmol})$, acetic acid $(0.2 \mathrm{~mL}, 3.5 \mathrm{mmol})$ and trimethylsilyl acetate $(0.2 \mathrm{~mL}, 1.3 \mathrm{mmol})$ at $-80{ }^{\circ} \mathrm{C}$. The solution was gradually warmed to $\mathrm{rt}$ and stirred overnight. The reaction mixture was quenched by the addition of water and extracted with dichloromethane. The organic phase was dried with $\mathrm{MgSO}_{4}$ and concentrated in vacuo. The crude mixture was purified by column chromatography ( $\mathrm{SiO}_{2}$, eluent: $20 \%$ ethyl acetate in hexane) to give $4 \mathrm{a}\left(51.8 \mathrm{mg}, 0.275 \mathrm{mmol}, 86 \%\right.$ yield). ${ }^{1} \mathrm{H}-\mathrm{NMR}$ $\left(600 \mathrm{MHz}, \mathrm{CDCl}_{3}\right) \delta 4.86(\mathrm{dt}, J=10.3,3.4 \mathrm{~Hz}, 1 \mathrm{H}), 3.68(\mathrm{~m}, 1 \mathrm{H}), 2.07(\mathrm{~s}, 3 \mathrm{H}), 1.75-1.23(\mathrm{~m}, 9 \mathrm{H})$, $0.92(\mathrm{t}, J=6.9 \mathrm{~Hz}, 3 \mathrm{H}), 0.90(\mathrm{t}, J=6.9 \mathrm{~Hz}, 3 \mathrm{H}) ;{ }^{13} \mathrm{C}-\mathrm{NMR}\left(150 \mathrm{MHz}, \mathrm{CDCl}_{3}\right) \delta 171.3,77.5,73.0$, $34.3,30.8,21.2,19.1,18.9,14.0,13.9$; HRMS (ESI-TOF) $m / z:[\mathrm{M}+\mathrm{Na}]^{+}$Calcd for $\mathrm{C}_{10} \mathrm{H}_{20} \mathrm{NaO}_{3}$ 211.1310; Found 211.1309.

Acetylation of $\mathbf{4 a}$ with acetic anhydride gave meso-4,5-diacetoxyoctane (5a) in $87 \%$ yield. Selected data for 5a: ${ }^{1} \mathrm{H}-\mathrm{NMR}\left(600 \mathrm{MHz}, \mathrm{CDCl}_{3}\right) \delta 4.98(\mathrm{~d}, J=11.0 \mathrm{~Hz}, 2 \mathrm{H}), 2.02(\mathrm{~s}, 6 \mathrm{H}), 1.54(\mathrm{~m}, 2 \mathrm{H}), 1.46$ $(\mathrm{m}, 2 \mathrm{H}), 1.36(\mathrm{~m}, 2 \mathrm{H}), 1.25(\mathrm{~m}, 2 \mathrm{H}), 0.89$ (t, $J=7.6 \mathrm{~Hz}, 6 \mathrm{H}) ;{ }^{13} \mathrm{C}-\mathrm{NMR}\left(150 \mathrm{MHz}, \mathrm{CDCl}_{3}\right) \delta 170.7$, 74.0, 31.3, 21.0, 18.8, 13.8. The NMR data agreed well with the reported values [72].

(4S,5S)-5-Hydroxyoctan-4-yl acetate $(4 S, 5 S)-\mathbf{4 b}$ : A Woodward reaction of trans-4-octene (1) was performed according to similar procedures described above and gave a racemic sample of $\mathbf{4 b}$ in $77 \%$ yield. ${ }^{1} \mathrm{H}-\mathrm{NMR}\left(600 \mathrm{MHz}, \mathrm{CDCl}_{3}\right) \delta 4.82(\mathrm{dt}, J=7.6,4.2 \mathrm{~Hz}, 1 \mathrm{H}), 3.57(\mathrm{~m}, 1 \mathrm{H}), 2.07$ (s, 3H), 1.63-1.27 $(\mathrm{m}, 8 \mathrm{H}), 0.90(\mathrm{t}, J=6.9 \mathrm{~Hz}, 3 \mathrm{H}) ;{ }^{13} \mathrm{C}-\mathrm{NMR}\left(150 \mathrm{MHz}, \mathrm{CDCl}_{3}\right) \delta 171.0,76.4,72.3,35.9,32.8,21.1$, $18.8,18.7,13.99,13.95$. The NMR data agreed well with the reported values [72]. HRMS (ESI-TOF) $m / z:[\mathrm{M}+\mathrm{Na}]^{+}$Calcd for $\mathrm{C}_{10} \mathrm{H}_{20} \mathrm{NaO}_{3} 211.1310$; Found 211.1303.

Enantioselective reaction of $\mathbf{1 b}(0.32 \mathrm{mmol})$ was performed using $\mathbf{7}$ and gave optically active $\mathbf{4 b}$ in $53 \%$ yield $(0.17 \mathrm{mmol})$. $[\alpha]_{\mathrm{D}}^{20}-22.2\left(c 0.90\right.$ in $\left.\mathrm{CHCl}_{3}\right)$. Ee of the product was determined to be $76 \%$ ee 
of $(4 S, 5 S)-4 \mathbf{b}$ by GC on a chiral stationary phase (DEX-CB, i.d. $0.25 \mathrm{~mm} \times 25 \mathrm{~m})$. Retention times of $(4 S, 5 S)-\mathbf{4 b}$ and $(4 R, 5 R)-\mathbf{4 b}$ were 7.8 and $8.2 \mathrm{~min}$, respectively, when the column temperature was maintained at $130{ }^{\circ} \mathrm{C}$.

Acetylation of $(4 S, 5 S)-\mathbf{4 b}$ with acetic anhydride gave the corresponding diacetoxy compound $(4 S, 5 S)-5 \mathbf{b}$ in $87 \%$ yield. Selected data for $(4 S, 5 S)-\mathbf{5 b}$ : ${ }^{1} \mathrm{H}-\mathrm{NMR}\left(600 \mathrm{MHz}, \mathrm{CDCl}_{3}\right) \delta 4.99(\mathrm{~m}, 2 \mathrm{H})$, $2.06(\mathrm{~s}, 6 \mathrm{H}), 1.53-1.24(\mathrm{~m}, 8 \mathrm{H}), 0.88(\mathrm{t}, J=6.9 \mathrm{~Hz}, 6 \mathrm{H}) ;{ }^{13} \mathrm{C}-\mathrm{NMR}\left(150 \mathrm{MHz}, \mathrm{CDCl}_{3}\right) \delta 170.7,73.7$, $32.9,20.9,18.5,13.9$. The NMR data agreed well with the reported values [73]. $[\alpha]_{\mathrm{D}}^{20}-42.1(c 0.96$ in $\left.\mathrm{CHCl}_{3}\right)$. Ee of the product was determined to be $76 \%$ ee of $(4 S, 5 S)-5 \mathbf{b}$ by GC on a chiral stationary phase (DEX-CB, i.d. $0.25 \mathrm{~mm} \times 25 \mathrm{~m})$. Retention times of $(4 S, 5 S)-5 \mathbf{b}$ and $(4 R, 5 R)-5 \mathbf{b}$ were 7.7 and 7.9 min, respectively, when the column temperature was maintained at $130{ }^{\circ} \mathrm{C}$.

The sample of (4S,5S)-5 $\mathbf{b}$ was hydrolyzed under basic conditions and octane-4,5-diol was obtained. Optical rotation of the diol obtained $\left([\alpha]_{\mathrm{D}}^{20}-33(c \quad 0.54\right.$ in EtOH $\left.)\right)$ indicates that the sample has $(4 S, 5 S)$-configuration; reported value for $(4 R, 5 R)$-octane-4,5-diol: [ $\alpha]_{\mathrm{D}}^{27}+44.4(c 0.12$ in EtOH) [74].

\section{Supplementary Materials}

Supplementary materials can be accessed at: http:/www.mdpi.com/1420-3049/20/09/17041/s1.

\section{Acknowledgments}

This research was partially supported by the Japan Society for the Promotion of Science (JSPS) through a Grant-in-Aid for Scientific Research (C) (23550059 and 26410057).

\section{Author Contributions}

M.S. and M.F. designed the project, and performed experiments. M.F. wrote the manuscript. T.S. helped with evaluation of the project.

\section{Conflicts of Interest}

The authors declare no conflict of interest.

\section{References and Notes}

1. Varvoglis, A. The Organic Chemistry of Polycoordinated Iodine; VCH: New York, NY, USA, 1992.

2. Varvoglis, A. Hypervalent Iodine in Organic Synthesis; Academic Press: San Diego, CA, USA, 1997.

3. Wirth, T. Hypervalent Iodine Chemistry; Springer: Berlin, Germany, 2003.

4. Zhdankin, V.V. Hypervalent Iodine Chemistry; John Wiley \& Sons: Chichester, UK, 2014.

5. Moriarty, R.M. Organohypervalent iodine: Development, applications, and future directions. J. Org. Chem. 2005, 70, 2893-2903.

6. Wirth, T. Hypervalent iodine chemistry in synthesis: Scope and new directions. Angew. Chem. Int. Ed. 2005, 44, 3656-3665.

7. Zhdankin, V.V.; Stang, P.J. Chemistry of polyvalent iodine. Chem. Rev. 2008, 108, 5299-5358.

8. Ochiai, M.; Miyamoto, K. Catalytic version of and reuse in hypervalent organo- $\lambda^{3}$ - and $-\lambda^{5}$-iodane oxidation. Eur. J. Org. Chem. 2008, 4229-4239, doi:10.1002/ejoc.200800416. 
9. Ngatimin, M.; Lupton, D.W. The discovery of catalytic enantioselective polyvalent iodine mediated reactions. Aust. J. Chem. 2010, 63, 653-658.

10. Liang, H.; Ciufolini, M.A. Chiral hypervalent iodine reagents in asymmetric reactions. Angew. Chem. Int. Ed. 2011, 50, 11849-11851.

11. Uyanik, M.; Ishihara, K. Conformationally-flexible chiral hypervalent organoiodine catalysts for enantioselective oxidative transformations. J. Synth. Org. Chem. Jpn. 2012, 70, 1116-1122.

12. Rawling, M.J.; Tomkinson, N.C.O. Metal-free syn-dioxygenation of alkenes. Org. Biomol. Chem. 2013, 11, 1434-1440.

13. Parra, A.; Reboredo, S. Chiral hypervalent iodine reagents: Synthesis and reactivity. Chem. Eur. J. 2013, 19, 17244-17260.

14. Brown, M.; Farid, U.; Wirth, T. Hypervalent iodine reagents as powerful electrophiles. Synlett 2013, 24, 424-431.

15. Dohi, T.; Kita, Y. New site-selective organoradical based on hypervalent iodine reagent for controlled alkane $\mathrm{sp}^{3} \mathrm{C}-\mathrm{H}$ oxidations. ChemCatChem 2014, 6, 76-78.

16. Singh, F.V.; Wirth, T. Hypervalent iodine-catalyzed oxidative functionalizations including stereoselective reactions. Chem. Asian J. 2014, 9, 950-971.

17. Romero, R.M.; Wöste, T.H.; Muñiz, K. Vicinal difunctionalization of alkenes with iodine(III) reagents and catalysts. Chem. Asian J. 2014, 9, 972-983.

18. Harned, A.M. Asymmetric oxidative dearomatizations promoted by hypervalent iodine(III) reagents: An opportunity for rational catalyst design? Tetrahedron Lett. 2014, 55, 4681-4689.

19. Berthiol, F. Reagent and catalyst design for asymmetric hypervalent iodine oxidations. Synthesis 2015, 47, 587-603.

20. Kang, Y.-B.; Gade, L.H. The nature of the catalytically active species in olefin dioxygenation with $\mathrm{PhI}(\mathrm{OAc}) 2$ : Metal or proton? J. Am. Chem. Soc. 2011, 133, 3658-3667.

21. Zhong, W.; Yang, J.; Meng, X.; Li, Z. BF 3 . OEt 2 -Promoted diastereoselective diacetoxylation of alkenes by $\mathrm{PhI}(\mathrm{OAc}) 2$. J. Org. Chem. 2011, 76, 9997-10004.

22. Li, Y.; Studer, A. Transition-metal-free trifluoromethylaminoxylation of alkenes. Angew. Chem. Int. Ed. 2012, 51, 8221-8224.

23. Nocquet-Thibault, S.; Retailleau, P.; Cariou, K.; Dodd, R.H. Iodine(III)-mediated umpolung of bromide salts for the ethoxybromination of enamides. Org. Lett. 2013, 15, 1842-1845.

24. Wang, Y.; Zhang, L.; Yang, Y.; Zhang, P.; Du, Z.; Wang, C. Alkene oxyalkylation enabled by merging rhenium catalysis with hypervalent iodine(III) reagents via decarboxylation. J. Am. Chem. Soc. 2013, 135, 18048-18051.

25. He, Y.-T.; Li, L.-H.; Yang, Y.-F.; Zhou, Z.-Z.; Hua, H.-L.; Liu, X.-Y.; Liang, Y.-M. Copper-catalyzed intermolecular cyanotrifluoromethylation of alkenes. Org. Lett. 2014, 16, 270-273.

26. Danneman, M.W.; Hong, K.B.; Johnston, J.N. Oxidative inter-/intermolecular alkene diamination of hydroxy styrenes with electron-rich amines. Org. Lett. 2015, 17, 2558-2561.

27. Lovick, H.M.; Michael, F.E. Metal-free highly regioselective aminotrifluoroacetoxylation of alkenes. J. Am. Chem. Soc. 2010, 132, 1249-1251.

28. Wardrop, D.J.; Bowen, E.G.; Forslund, R.E.; Sussman, A.D.; Weerasekera, S.L. Intramolecular oxamidation of unsaturated $O$-alkyl hydroxamates: A remarkably versatile entry to hydroxy lactams. J. Am. Chem. Soc. 2010, 132, 1188-1189. 
29. Karila, D.; Leman, L.; Dodd, R.H. Copper-catalyzed iminoiodane-mediated aminolactonization of olefins: Application to the synthesis of 5,5-disubstituted butyrolactones. Org. Lett. 2011, 13, 5830-5833.

30. Wei, H.-L.; Piou, T.; Dufour, J.; Neuville, L.; Zhu, J. Iodo-carbocyclization of electron-deficient alkenes: Synthesis of oxindoles and spirooxindoles. Org. Lett. 2011, 13, 2244-2247.

31. Zhu, R.; Buchwald, S.L. Copper-catalyzed oxytrifluoromethylation of unactivated alkenes. J. Am. Chem. Soc. 2012, 134, 12462-12465.

32. Tu, D.; Ma, L.; Tong, X.; Deng, X.; Xia, C. Synthesis of pyrrolo[2,3-b]indole via iodine(III)-mediated intramolecular annulation. Org. Lett. 2012, 14, 4830-4833.

33. Zheng, Y.; Li, X.; Ren, C.; Zhang-Negrerie, D.; Du, Y.; Zhao, K. Synthesis of oxazoles from enamides via phenyliodine diacetate-mediated intramolecular oxidative cyclization. J. Org. Chem. 2012, 77, 10353-10361.

34. Fujita, M.; Mori, K.; Shimogaki, M.; Sugimura, T. Asymmetric synthesis of 4,8-dihydroxyisochroman1-one polyketide metabolites using chiral hypervalent iodine(III). Org. Lett. 2012, 14, 1294-1297.

35. Zhu, R.; Buchwald, S.L. Enantioselective functionalization of radical intermediates in redox catalysis: Copper-catalyzed asymmetric oxytrifluoromethylation of alkenes. Angew. Chem. Int. Ed. 2013, 52, 12655-12658.

36. Fujita, M.; Mori, K.; Shimogaki, M.; Sugimura, T. Total synthesis of (12R)- and (12S)-12hydroxymonocerins: Stereoselective oxylactonization using a chiral hypervalent iodine(III) species. RSC Adv. 2013, 3, 17717-17725.

37. Li, L.; Deng, M.; Zheng, S.-C.; Xiong, Y.-P.; Tan, B.; Liu, X.-Y. Metal-free direct intramolecular carbotrifluoromethylation of alkenes to functionalized trifluoromethyl azaheterocycles. Org. Lett. 2014, 16, 504-507.

38. Chen, H.; Kaga, A.; Chiba, S. Diastereoselective aminooxygenation and diamination of alkenes with amidines by hypervalent iodine(III) reagents. Org. Lett. 2014, 16, 6136-6139.

39. Takesue, T.; Fujita, M.; Sugimura, T.; Akutsu, H. A series of two oxidation reactions of orthoalkenylbenzamide with hypervalent iodine(III): A concise entry into $(3 R, 4 R)-4$-hydroxymellein and (3R,4R)-4-hydroxy-6-methoxymellein. Org. Lett. 2014, 16, 4634-4637.

40. Alhalib, A.; Kamouka, S.; Moran, W.J. Iodoarene-catalyzed cyclizations of unsaturated amides. Org. Lett. 2015, 17, 1453-1456.

41. Steuff, J.; Hövelmann, C.H.; Nieger, M.; Muñiz, K. Palladium(II)-catalyzed intramolecular diamination of unfunctionalized alkenes. J. Am. Chem. Soc. 2005, 127, 14586-14587.

42. Muñiz, K.; Hövelmann, C.H.; Steuff, J. Oxidative diamination of alkenes with ureas as nitrogen sources: Mechanistic pathways in the presence of a high oxidation state palladium catalyst. J. Am. Chem. Soc. 2008, 130, 763-773.

43. Cochran, B.M.; Michael, F.E. Metal-free oxidative cyclization of urea-tethered alkenes with hypervalent iodine. Org. Lett. 2008, 10, 5039-5042.

44. Mizar, P.; Laverny, A.; El-Sherbini, M.; Farid, U.; Brown, M.; Malmedy, F.; Wirth, T. Enantioselective diamination with novel chiral hypervalent iodine catalysts. Chem. Eur. J. 2014, 20, 9910-9913.

45. Anumandla, D.; Littlefield, R.; Jeffrey, C.S. Oxidative 1,4-diamination of dienes using simple urea derivatives. Org. Lett. 2014, 16, 5112-5115. 
46. Karade, N.N.; Shirodkar, S.G.; Patil, M.N.; Potrelar, R.A.; Larade, H.N. Diacetoxyiodobenzenemediated oxidative addition of 1,3-dicarbonyl compounds to olefins: An efficient one-pot synthesis of 2,3-dihydrofuran derivatives. Tetrahedron Lett. 2003, 44, 6729-6731.

47. Yoshimura, A.; Middleton, K.R.; Todora, A.D.; Kastern, B.J.; Koski, S.R.; Maskaev, A.V.; Zhdankin, V.V. Hypervalent iodine catalyzed generation of nitrile oxides from oximes and their cycloaddition with alkenes or alkynes. Org. Lett. 2013, 15, 4010-4013.

48. Pittman, C.U., Jr.; McManus, S.P.; Larsen, J.W. 1,3-Dioxolan-2-ylium and related heterocyclic cations. Chem. Rev. 1972, 72, 357-438.

49. Lorenz, W.; Maas, G. O-Acylation of $\alpha$-diazo ketones. A novel route to alkenediazonium and 1,3-dioxolium salts. J. Org. Chem. 1987, 52, 375-381.

50. Prévost, C. Sur un complexe iodo-argento-benzoïque et son application à l'oxydation des combinaisons éthyléniques en $\alpha$-glycols. Comptes Rendus 1933, 196, 1129-1131.

51. Woodward, R.B.; Brutcher, F.V., Jr. cis-Hydroxylation of a synthetic steroid intermediate with iodine, silver acetate and wet acetic acid. J. Am. Chem. Soc. 1958, 80, 209-211.

52. Zhdankin, V.V.; Tykwinski, R.; Berglund, B.; Mullikin, M.; Caple, R.; Zefirov, N.S.; Koz'min, A.S. Iodosobenzene tetrafluoroborate, hexafluoroantimonate, and hexafluorophosphate: Stable electrophilic hypervalent iodine reagents without nucleophilic ligands. J. Org. Chem. 1989, $54,2609-2612$.

53. Ochiai, M.; Miyamoto, K.; Shiro, M.; Ozawa, T.; Yamaguchi, K. Isolation, characterization, and reaction of activated iodosylbenzene monomer hydroxy(phenyl)iodonium ion with hypervalent bonding: Supramolecular complex $\mathrm{PhI}^{+} \mathrm{OH} \cdot 18$-crown-6 with secondary $\mathrm{I} \cdots \mathrm{O}$ interactions. $J . A m$. Chem. Soc. 2003, 125, 13006-13007.

54. Emmanuvel, L.; Shaikh, T.M.A.; Sudalai, A. NaIO4/LiBr-mediated diastereoselective dihydroxylation of olefins: A catalytic approach to the Prevost-Woodward reaction. Org. Lett. 2005, 7, 5071-5074.

55. Li, Y.; Song, D.; Dong, V.M. Palladium-catalyzed olefin dioxygenation. J. Am. Chem. Soc. 2008, 130, 2962-2964.

56. Seayad, J.; Seayad, A.M.; Chai, C.L.L. Copper-catalyzed diacetoxylation of olefins using PhI(OAc)2 as oxidant. Org. Lett. 2010, 12, 1412-1415.

57. Fujita, M.; Wakita, M.; Sugimura, T. Enantioselective Prévost and Woodward reactions using chiral hypervalent iodine(III): Switchover of stereochemical course of an optically active 1,3-dioxolan-2-yl cation. Chem. Commun. 2011, 47, 3983-3985.

58. Zhong, W.; Liu, S.; Yang, J.; Meng, X.; Li, Z. Metal-free, organocatalytic syn diacetoxylation of alkenes. Org. Lett. 2012, 14, 3336-3339.

59. Mukaiyama, T.; Hayashi, Y.; Hashimoto, Y. Regioselective alkylation of 1,3-dioxolan-2-ylium cation derived from $\alpha, \beta$-unsaturated aldehyde ethylene acetal with lithium organo compounds. Chem. Lett. 1986, 1627-1630, doi:10.1246/cl.1986.1627.

60. Hayashi, Y.; Wariishi, K.; Mukaiyama, T. Oxidative carbon-carbon bond forming reaction via a 1,3-dioxolan-2-ylium cation. Chem. Lett. 1987, 1243-1246.

61. An enamine nucleophile, 4-(1-phenylvinyl)morpholine was used instead of $\mathbf{2 d}$ in the reaction of 1b. However, no 3n was observed. 
62. Fujita, M.; Okuno, S.; Lee, H.J.; Sugimura, T.; Okuyama, T. Enantiodifferentiating tetrahydrofuranylation of but-3-enyl carboxylates using optically active hypervalent iodine(III) reagents via a 1,3-dioxan-2-yl cation intermediate. Tetrahedron Lett. 2007, 48, 8691-8694.

63. Uyanik, M.; Yasui, T.; Ishihara, K. Enantioselective Kita oxidative spirolactonization catalyzed by in situ generated chiral hypervalent iodine(III) species. Angew. Chem. Int. Ed. 2010, 49, 2175-2177.

64. Fujita, M.; Yoshida, Y.; Miyata, K.; Wakisaka, A.; Sugimura, T. Enantiodifferentiating endo-selective oxylactonization of ortho-alk-1-enylbenzoate with a lactate-derived aryl- $\lambda^{3}$-iodane. Angew. Chem. Int. Ed. 2010, 49, 7068-7071.

65. Uyanik, M.; Yasui, T.; Ishihara, K. Chiral hypervalent iodine-catalyzed enantioselective oxidative Kita spirolactonization of 1-naphthol derivatives and one-pot diastereo-selective oxidation to epoxyspirolactones. Tetrahedron 2010, 66, 5841-5851.

66. Röben, C.; Souto, J.A.; González, Y.; Lishchynskyi, A.; Muñiz, K. Enantioselective metal-free diamination of styrenes. Angew. Chem. Int. Ed. 2011, 50, 9478-9482.

67. Farid, U.; Wirth, T. Highly stereoselective metal-free oxyaminations using chiral hypervalent iodine reagents. Angew. Chem. Int. Ed. 2012, 51, 3462-3465.

68. Kong, W.; Feige, P.; de Haro, T.; Nevado, C. Regio- and enantioselective aminofluorination of alkenes. Angew. Chem. Int. Ed. 2013, 52, 2469-2473.

69. Farid, U.; Malmedy, F.; Claveau, R.; Albers, L.; Wirth, T. Stereoselective rearrangements with chiral hypervalent iodine reagents. Angew. Chem. Int. Ed. 2013, 52, 7018-7022.

70. Shimogaki, M.; Fujita, M.; Sugimura, T. Enantioselective oxidation of alkenylbenzoates catalyzed by chiral hypervalent iodine(III) to yield 4-hydroxyisochroman-1-ones. Eur. J. Org. Chem. 2013, 2013, 7128-7138.

71. Wu, H.; He, Y.-P.; Xu, L.; Zhang, D.-Y.; Gong, L.-Z. Asymmetric organocatalytic direct $\mathrm{C}\left(\mathrm{sp}^{2}\right)-\mathrm{H} / \mathrm{C}\left(\mathrm{sp}^{3}\right)-\mathrm{H}$ oxidative cross-coupling by chiral iodine reagents. Angew. Chem. Int. Ed. 2014, 53, 3466-3469.

72. Lethbridge, A.; Norman, R.O.C.; Thomas, C.B.; Parr, W.J.E. Oxidation of oct-1-ene and trans-oct-4-ene by lead(IV), thallium(III), and mercury(II) acetates. J. Chem. Soc. Perkin Trans. 1975, 1, 231-241.

73. Uemura, S.; Ohe, K.; Fukuzawa, S.-I.; Patil, S.R.; Sugita, N. Dominant cis-diacetoxylation of alkenes with tellurium(IV) oxide and lithium bromide in acetic acid. J. Organomet. Chem. 1986, 316, 67-78.

74. Fujioka, H.; Matsunaga, N.; Kitagawa, H.; Nagatomi, Y.; Kondo, M.; Kita, Y. Asymmetric synthesis using $\mathrm{C}_{2}$-symmetric diols: Use of (5R,6R)-3-acetoxy-5,6-diphenyl-1,4-dioxan-2-one as a chiral synthetic equivalent of 1,2-ethanediol 1,2-dicarbocation. Tetrahedron Asymmetry 1995, 6, 2117-2020.

Sample Availability: Not available.

(C) 2015 by the authors; licensee MDPI, Basel, Switzerland. This article is an open access article distributed under the terms and conditions of the Creative Commons Attribution license (http://creativecommons.org/licenses/by/4.0/). 